\title{
Plasma heating in solar flares and their soft and hard X-ray emissions
}

\author{
R. Falewicz \\ Astronomical Institute, University of Wrocław, 51-622 Wrocław, ul. Kopernika 11, Poland \\ falewicz@astro.uni.wroc.pl
}

\begin{abstract}
In this paper, the energy budgets of two single-loop like flares observed in X-ray are analysed under the assumption that non-thermal electrons (NTEs) are the only source of plasma heating during all phases of both events. The flares were observed by RHESSI and GOES on February $20^{\text {th }}, 2002$ and June $2^{\text {nd }}, 2002$, respectively. Using a 1D hydrodynamic code for both flares the energy deposited in the chromosphere was derived applying RHESSI observational data. The use of the Fokker-Planck formalism permits the calculation of distributions of the non-thermal electrons in flaring loops, thus spatial distributions of the X-ray non-thermal emissions and integral fluxes for the selected energy ranges which were compared with the observed ones. Additionally, a comparative analysis of the spatial distributions of the signals in the RHESSI images was conducted for the footpoints and for the entire flare loops in selected energy ranges with these quantities fluxes obtained from the models. The best compatibility of the model and observations was obtained for the June $2^{\text {nd }}, 2002$ event in the $0.5-4 \AA$ GOES range and total fluxes in the 6-12 keV, $12-25 \mathrm{keV}, 20-25 \mathrm{keV}$ and $50-100 \mathrm{keV}$ energy bands. Results of photometry of the individual flaring structures in a high energy range shows that the best compliance occurred for the June $2^{\text {nd }}, 2002$ flare, where the synthesized emissions were $30 \%$ or more higher than the observed emissions. For the February $20^{\text {th }}, 2002$ flare, synthesized emission is about 4 times lower than the observed one. However, in the low energy range the best conformity was obtained for the February $20^{\text {th }}, 2002$ flare, where emission from the model is about $11 \%$ lower than the observed one. The larger inconsistency occurs for the June $2^{\text {nd }}, 2002$ solar flare, where synthesized emission is about 12 times greater or even more than the observed emission. Some part of these differences may be caused by inevitable flaws of the applied methodology, like by an assumption that the model of the flare is symmetric and there are no differences in the emissions originating from the feet of the flares loop and by relative simplicity of the applied numerical 1D code and procedures. No doubt a significant refinement of the applied numerical models and more sophisticated implementation of the various physical mechanisms involved are required to achieve
\end{abstract}


a better agreement. Despite these problems, a collation of modelled results with observations shows that soft and hard X-ray emissions observed for analysed single-loop like events may be fully explained by electron beam-driven evaporation only.

Subject headings: Sun: flares — Sun: X-rays, gamma rays - Sun: corona - Sun: chromosphere

\section{Introduction}

Despite the significant progress already achieved both in observational and theoretical investigations as well as in numerical modelling of solar flares, the main physical processes involved in energy conversion, transfer, deposition and losses in solar flares are only understand fragmentarily. However, broad insight is crucial for solving the most important questions, like a balance of energy in the solar flares, a prediction of solar flare magnitudes and their emission spectra, and emission of high energy particles. It is commonly accepted that during the impulsive phase of solar flares as well as to some extent, before and after the impulsive phase, beams of non-thermal electrons, accelerated somewhere in the solar corona stream along magnetic field lines toward the chromosphere, where they heat by collision the dense matter near the feet of the loops. The interactions of the non-thermal electrons with dense matter also cause a strong and variable in time emission of hard X-rays (HXR) called electron-ion bremsstrahlung which was described by Brown (1971) and Emslie (1978). The chromospheric matter, heated up to coronal temperatures, expands and fills the magnetic ropes (such a process is called chromospheric evaporation) and it mainly emits soft X-rays (SXR) and also some thermal hard Xrays (Antonucci, Gabriel, \& Dennis 1984; Fisher, Canfield, \& McClymont 1985b). Taking into account that the main physical processes occurring in solar flares are very complicated and crossrelated and very difficult in comprehensive analytical description, a numerical modelling of the flares based on their observational properties allows us to extend our understanding of the physical aspects of the flares. The first numerical models explored the hydrodynamic response of the solar atmosphere to heating by electron beams (Nagai \& Emslie 1984; MacNeice et al. 1984; Mariska \& Poland 1985; Fisher, Canfield. \& McClymont 1985a,b.c; Mariska. Emslie. \& Li 1989; Serio et al. 1991). Reale et al. (1997) derived the method of estimation of the sizes of spatially unresolved solar and stellar coronal flaring structures based on the X-ray light curves and timeresolved temperature and emission measure values measured during flare decay, applying hydrodynamic modelling. More sophisticated multi-thread, time-dependent hydrodynamic simulations of solar flares was applied by Warren (2006) to observations of the Masuda's flare of January $13^{\text {th }}$, 1992. Combined modelling of acceleration, transport, and hydrodynamic response in solar flares was described in the paper by Liu, Petrosian, \& Mariska (2009). They tested three models of solar 
flare heating, repeating the same simulation with two kinds of injection downward-beamed electrons using different methods of particle transport and the new heating rates. As a main result of this comparison, the model calculated using analytical approximation of particle transport gives about $10 \%$ difference compared to the model calculated with the Fokker-Planck formalism but provides an acceptable approximation. In addition, it was found that using the model with the Fokker-Planck description of the particle transport and if the injected electron spectrum is based on stochastic acceleration, the authors found higher coronal temperatures and densities, larger upflow velocities, and faster increases of these quantities than in the model with electron injection of power law. Falewicz, Rudawy, \& Siarkowski (2009a) using hydrodynamic modelling examined the sensitivity of the GOES classification to the non-thermal electron beam properties using Yohkoh data, while a similar analysis was performed for the RHESSI data by Reep, Bradshaw, \& McAteer (2013).

In this paper we used all achievements of our previous investigations of the energy transfer and deposition processes in simple, single-loop solar flares, in particular our investigations of interactions of the non-thermal electrons with matter (Falewicz, Rudawy, \& Siarkowski 2009a,b; Falewicz, Siarkowski, \& Rudawy 2011; Siarkowski, Falewicz, \& Rudawy 2009) and numerical modelling of the X-ray flare emission (Falewicz, Rudawy, \& Siarkowski 2009b; Falewicz, Siarkowski, \& Rudawy 2011). Additionally, in our work we took into account the results presented by Liu. Petrosian, \& Mariska (2009), which validated certain simplifications during the modelling of solar flares, and were helpful in the interpretation of the results. We applied an essentially modified by us hydrodynamic numerical code of the Naval Research Laboratory (NRL), see Mariska et al. (1982); Mariska, Emslie, \& Li (1989) and Falewicz, Rudawy, \& Siarkowski (2009a) where a detailed description of the modified code is given. We modelled a heating process of the flaring plasma using Fisher's approximation (Fisher 1989) on the basis of energy flux parameters evaluated using observed HXR spectra. In the present paper we apply the Fokker-Planck formalism of the electron precipitation process through the plasma in the loops following method already evaluated by McTiernan \& Petrosian (1990). We computed the distributions of the non-thermal electrons in a flaring loop, spatial distributions of the X-ray non-thermal emissions and integral fluxes for the selected energy ranges which were compared with the observed fluxes in relevant energy ranges. Additionally, we also conducted a comparative analysis of the spatial distribution of the signal in the RHESSI images for the footpoints and entire flare loops in selected energy ranges, and compared these quantities with the fluxes obtained from the models. A conformity of fluxes was applied as a measure of the quality of the calculated flare models and applicability of used procedures and simplifications.

In this paper we present the results of the numerical modelling of two solar single-loop like flares. Section 2 describes the analysed events. Section 3 shows the details of the HXR spectra fitting procedure, numerical modelling algorithm and methods of calculation of thermal and non- 
thermal emissions. Section 4 presents the obtained results. The conclusions are discussed in Section 5.

\section{Observational data}

For analysis we selected solar flares which look like single-loop structures in X-rays, best for applied numerical model. The found flares were observed on February $20^{\text {th }}, 2002$, and June $2^{\text {nd }}, 2002$. Both events were presented and analysed in several papers. The emissions of the flares were registered by the Reuven Ramaty High Energy Solar Spectroscopic Imager (RHESSI) satellite (Lin et al. 2002). The RHESSI was designed to image solar flares in photons having energies from soft X-rays (3 keV) to gamma rays $(17 \mathrm{MeV})$ with a high temporal and energy resolution and also to provide high resolution spectroscopy in the same energy range. The RHESSI has nine coaxial germanium detectors with high signal sensitivity and allows a restoration of the images and spectra which are very useful for investigation of the non-thermal emission of solar flares. In case of excessively high photon fluxes, RHESSI automatically limits the readings using attenuators, unfortunately inserting also troublesome discontinuities of the recorded data (Lin et al. 2002; Hurford et al. 2002; Smith et al. 2002). However, the both investigated flares were observed with the A1 attenuator permanently on. GOES X-ray data were also used. The GOES (Geostationary Operational Environmental Satellites) satellites since 1974 have continuously recorded integrated solar X-ray fluxes in two energy bands 1-8 $\AA$ and 0.5-4 $\AA$ with $3 \mathrm{~s}$ temporal resolution (Donnelly, Grubb, \& Cowley 1977). To confirm the likely single loop structure of the analysed flares, additional observations from the Extreme-ultraviolet Imaging Telescope (EIT) were also used. EIT was installed on board the Solar and Heliospheric Observatory (SOHO; Delaboudinier̀e et al. 1995) and provides full-disk images taken in four bands: $171 \AA$ A, $195 \AA$, 284 $\AA$, and $304 \AA$ with 5 arcsec spatial resolution.

\subsection{February $20^{\text {th }}, \mathbf{2 0 0 2}$ solar flare}

This event was discussed already by Aschwanden, Brown, \& Kontar (2002) studying the height at which the HXR emission originates, by Sui et al. (2002) modelling the HXR emission, and by Krucker \& Lin (2002) who investigated the relationship between the brightnesses of the footpoints. Veronig et al. (2005) studied physics of the Neupert effect and Guo et al. (2011) presented a new method to study in detail the temporal evolution of thermal and non-thermal photon fluxes applied to this flare.

The C7.5 GOES class flare occurred in active region NOAA 9825 on February $20^{\text {th }}, 2002$ 


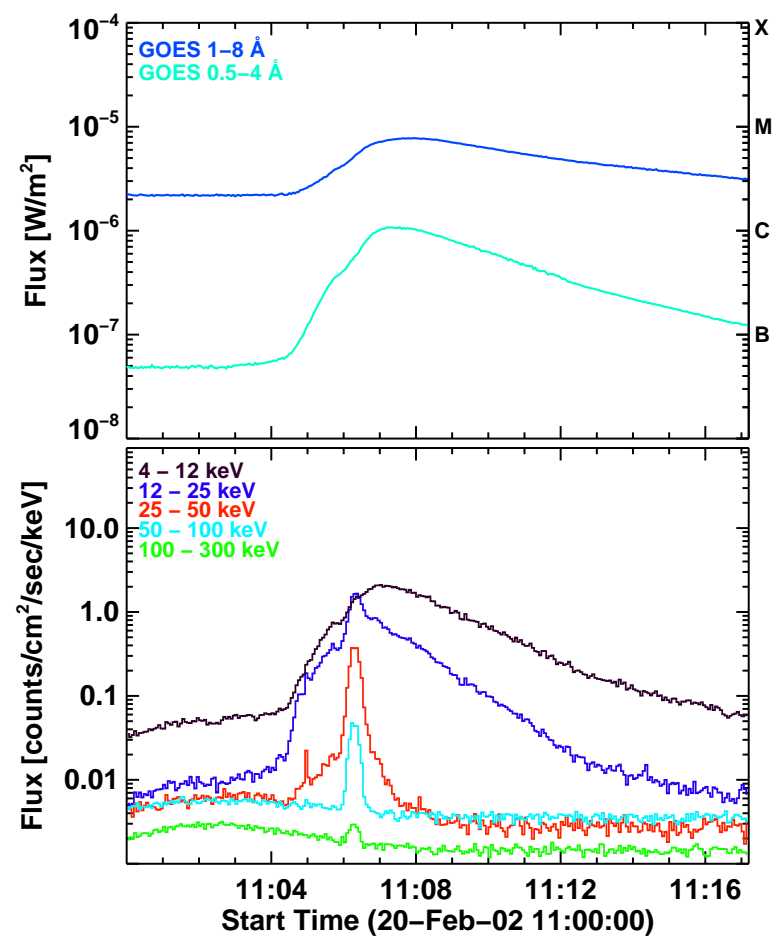

Fig. 1.- GOES X-ray 0.5-4 $\AA$ and 1-8 $\AA$ light curves (upper panel) and RHESSI light curves of five energy bands between 4 and $300 \mathrm{keV}$ (lower panel) taken during the C7.5 GOES class solar flare on February $20^{\text {th }}, 2002$.

which was located near the western limb of the solar disk. In this region on that day, before the start of the analysed flare four events occurred, M4.2 (02:44), M5.1 (05:52), C2.5 (07:41), M4.3 (09:46) and afterwards two C-class C4.5 (11:29) and C2.9 (15:59). Seven flares were observed in the active region on that day. The SXR (1-8 $\AA$ ) emission of the analysed flare recorded by GOES started at 11:02 UT, reached its maximum at 11:07 UT, and was observed up to 11:29 UT. A harder emission recorded by GOES (0.5-4 $\AA$ ) started to increase and peaked thereabouts at the same time as the softer flux (1-8 $\AA$ ). GOES light curves of the analysed flare are presented in Figure 1 (upper panel). RHESSI X-ray light curves of the flare taken in five energy bands are shown in Figure 1 (lower panel). The impulsive phase of the flare registered by RHESSI in X-rays above $25 \mathrm{keV}$ started at 11:04:28 UT, had its maximum at 11:06:08 UT and was observed even in the energy range of 100-300 keV. The SXR emission registered by RHESSI below $12 \mathrm{keV}$ started to rise simultaneously with GOES 0.5-4 $\AA$ emission and the time course of the fluxes looks very similar.

Images of the flare were reconstructed using RHESSI data gathered with sub-collimators $3 \mathrm{~F}$, 4F, 5F, 6F, 8F and 9F integrated over $8 \mathrm{sec}$ periods using PIXON imaging algorithm with a 1 arcsec pixel size (Metcalf et al. 1996; Hurford et al. 2002). The images restored for the low energy band 6-12 keV (see Figure 2, left panel) show a single source located between two footpoints visible in 

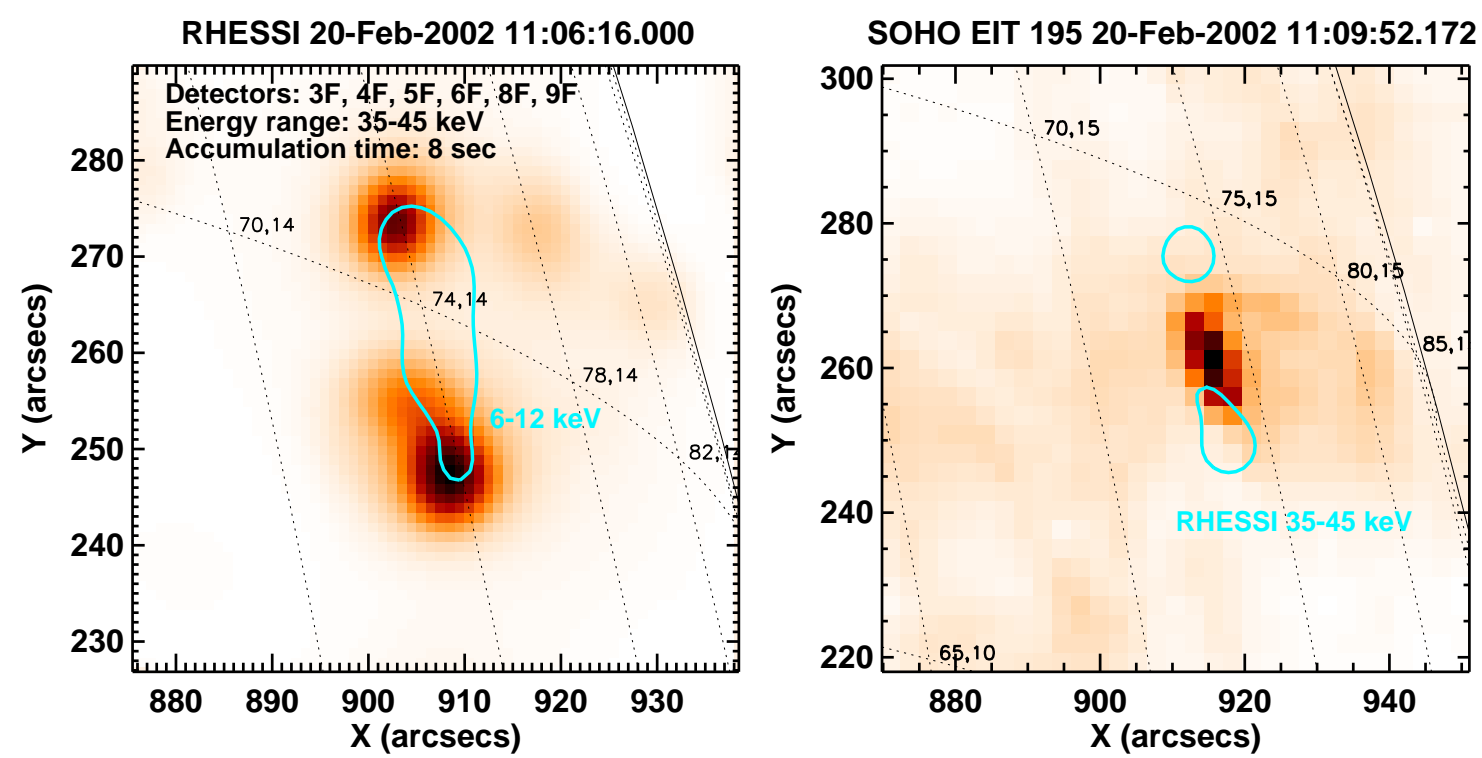

Fig. 2.- Images of the C7.5 GOES class solar flare on the February 20 ${ }^{\text {th }}$, 2002. Left panel: an image restored using the PIXON method in 35-45 keV energy band. The signal was accumulated between 11:06:16 UT and 11:06:24 UT. Right panel: SOHO/EIT $195 \AA$ image taken at 11:09:52 UT (grey scale) overplotted with the RHESSI 35-45 keV image registered at 11:06:16 UT (contours).

higher energy (35-45 keV). The image recorded by SOHO/EIT $195 \AA$ at 11:06:15 UT (see Figure 2 right panel) about four minutes later, indicates a structure very similar to single source recorded by RHESSI in 6-9 keV. Hard X-ray images were used to determine the main geometrical parameters of the flaring loop using a method proposed by Aschwanden et al. (1999). The cross-section of the loop $S=(7.16 \pm 6.83) \times 10^{16} \mathrm{~cm}^{2}$ was estimated as an average area of both footpoints delimited with isophotes of $30 \%$ of the maximum flux in the $35-45 \mathrm{keV}$ energy range. The cross-section of flaring loops of both analysed events was assumed to be constant. Half-length of the loop $L_{0}=(1.63 \pm 0.11) \times 10^{9} \mathrm{~cm}$ was estimated from a distance between the centres of gravity of the footpoints, assuming a semi-circular shape of the loop. Samples of RHESSI spectra taken before, during, and after the impulsive phase of the February $20^{\text {th }}, 2002$ flare and fitted with the single temperature thermal model and thick-target model are shown in Figure 3.

\subsection{June $2^{\text {nd }}, 2002$ solar flare}

The second analysed event was already described and analysed by Krucker \& Lin (2002) who investigated the relationship between the brightnesses of the footpoints. Veronig et al. (2005) analysed this flare in their study of physics of the Neupert effect. In papers by Sui, Holman, \& Dennis 

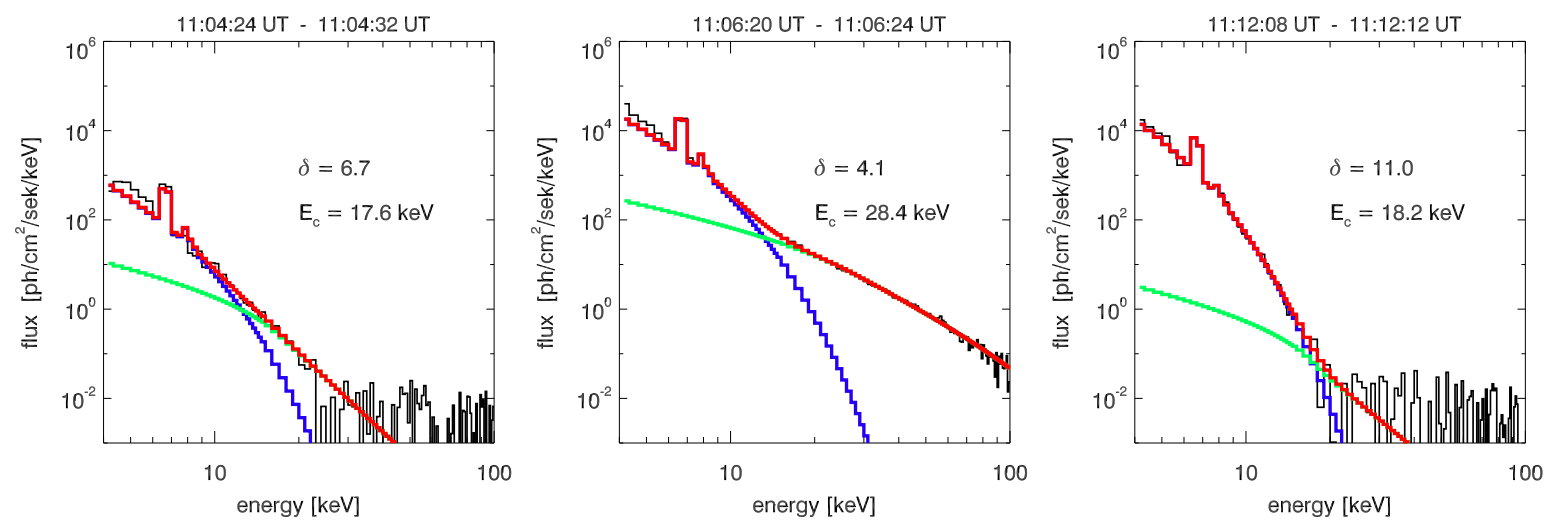

Fig. 3.- RHESSI spectra taken before (left panel), during (middle panel), and after the impulsive phase (right panel) of the February $20^{\text {th }}, 2002$ flare. The spectra were fitted with the single temperature thermal model (blue colour) and thick-target model (green). The summed fits of the spectra are shown in red. The obtained values of the power-law index of the electron energy distribution $\delta$, and low energy cut-off of the electron distribution $\mathrm{E}_{\mathrm{c}}$ were determined on the basis of the thick-target model and used as characteristics of the injected electrons in the model.

(2006, 2008) the authors discussed the morphology and evolution of the flare, particularly in the EUV and $\mathrm{H} \alpha$ energy range. They showed evidence for multiple-loop interactions as the cause of the flare. Also Meshalkina et al. (2009) examined various scenarios of the flare and proposed a three-dimensional scheme in which the filament eruption and flare were caused by interaction of the magnetic ropes.

The investigated flare occurred in active region (AR) NOAA 9973 (S17E23) on June $2^{\text {nd }}$, 2002. It was classified as a C9.4 GOES class flare. A magnetic class of the active region was beta-gamma and it produced three $\mathrm{C}$ class flares on that day, including the analysed event. The GOES 1-8 ^ flux started to increase slowly at 11:41 UT, reached a maximum at 11:47 UT, and was observed up to 11:55 UT. The time course of its SXR emission is not typical, having a flat maximum particularly visible in harder energy band recorded by GOES (0.5-4 $)$ ). GOES X-ray light curves of the flare are shown in Figure 4 (upper panel). RHESSI X-ray light curves of the flare taken in five energy bands are shown in Figure 4 (lower panel). The impulsive phase started in X-rays above $25 \mathrm{keV}$ at 11:44:14 UT, had double maximum at 11:44:34 and 11:44:54 UT, and was observed up to 11:46:02 UT. The hard X-ray emission of the impulsive phase was observed up to $300 \mathrm{keV}$. The X-ray emission below $25 \mathrm{keV}$ started to rise simultaneously with the GOES emission. Small increases of emissions causing flattening of the SXR maxima occurred at the same time in the GOES emission and RHESSI channels 4-12 keV and 12-25 keV at 11:47:42 UT.

The start of the impulsive phase occurred simultaneously with an increase of the soft X-rays emissions and no preheating prior to the impulsive phase was observed. 


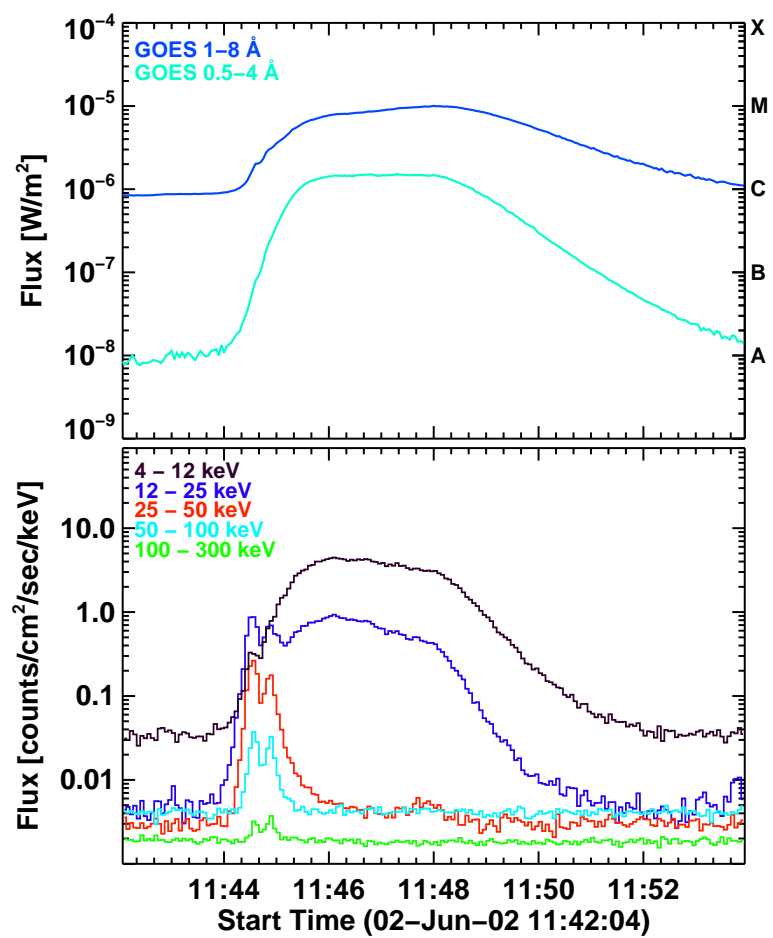

Fig. 4.- GOES X-ray 0.5-4 $\AA$ and 1-8 $\AA$ light curves (upper panel) and RHESSI light curves of five energy bands between 4 and $300 \mathrm{keV}$ (lower panel) taken during the C9.4 GOES class solar flare on June $2^{\text {nd }}, 2002$.

The images of the flare were obtained using RHESSI data collected with sub-collimators 3F, $4 \mathrm{~F}, 5 \mathrm{~F}, 6 \mathrm{~F}, 8 \mathrm{~F}$ and $9 \mathrm{~F}$ integrated over $48 \mathrm{~s}$ periods and the PIXON imaging algorithm with 1 arcsec pixel size. The image restored in the $30-80 \mathrm{keV}$ energy range revealed two distinct footpoints, where the western footpoint is brighter than the eastern (Figure 5 left panel). In the 6-12 keV energy range the entire flare loop was seen. The image recorded by SOHO/EIT $195 \AA$ at 11:50:54 UT, after the impulsive phase of the flare and overplotted with the RHESSI 30-80 keV PIXON image (contour) registered at 11:44:20 UT is shown in Figure 5 right panel. The main flare loop visible in the EUV range spatially coincides well with footpoints visible in the RHESSI 30-80 keV range. A method of determination of the geometrical parameters was the same as for the first flare. The cross section of the loop $S=(6.04 \pm 5.60) \times 10^{16} \mathrm{~cm}^{2}$ was estimated as an average area of the feet delimited with isophote of $30 \%$ of the maximum flux in the $30-80 \mathrm{keV}$ energy range. Halflength of the loop was estimated as $L_{0}=(8.37 \pm 1.13) \times 10^{8} \mathrm{~cm}$. Samples of RHESSI spectra taken before, during and after the impulsive phase are shown in Figure 6. 

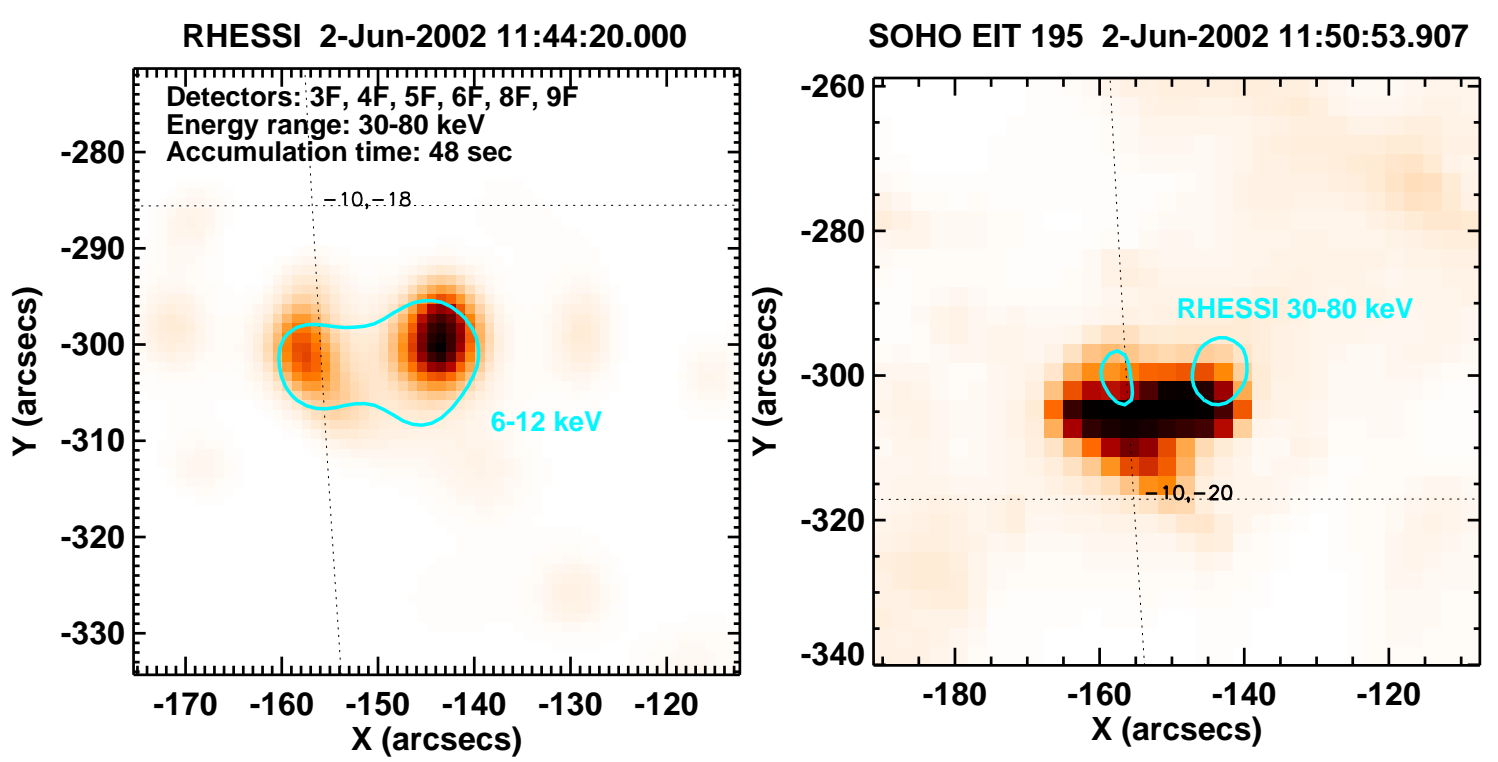

Fig. 5.- Images of the C9.4 GOES class solar flare on the June $2^{\text {nd }}, 2002$. Left panel: an image restored using the PIXON method in the $30-80 \mathrm{keV}$ energy band, the signal was accumulated between 11:44:20 UT and 11:45:08 UT, at the maximum of the impulsive phase (grey scale) overplotted with the RHESSI 6-9 keV image (contour). Right panel: SOHO/EIT $195 \AA$ image taken at 11:50:54 UT, after the impulsive phase of the flare, overplotted with the RHESSI $30-80 \mathrm{keV}$ PIXON image (contour) registered at 11:44:20 UT.

\section{Calculations}

The procedure of the numerical modelling used in this paper is very similar to the one described in previous publications (Siarkowski. Falewicz, \& Rudawy 2009; Falewicz. Siarkowski, \& Rudawy 2011). The main assumption of the method is that interactions of the non-thermal electrons (NTEs) with plasma are the only source of plasma heating during all phases of the analysed events. The same hydrodynamic model of the analysed flares was used, where the non-thermal electron beams derived from RHESSI spectra as the carriers of heating energy deposited, via Coulomb collisions. The energy deposition rates were calculated using an approximation given by Fisher (1989). As in the previous paper (Falewicz, Siarkowski, \& Rudawy 2011) the low energy cut-off of the electron distribution $\mathrm{E}_{\mathrm{c}}$ was automatically optimised by comparison at each time step of the observed SXR GOES flux in the 1-8 $\AA$ range and the calculated one. In this paper in addition, for each time step the steady-state electron spatial and spectral distributions in the loop were calculated using a Fokker-Planck code (McTiernan \& Petrosian 1990). Known distributions of the non-thermal electrons and physical parameters of the plasma along the flaring loop allowed for the calculation of spatial distributions of the X-ray thermal and non-thermal emissions, to derive integral fluxes in the selected energy ranges and to compare them with the fluxes observed in the same energy ranges 

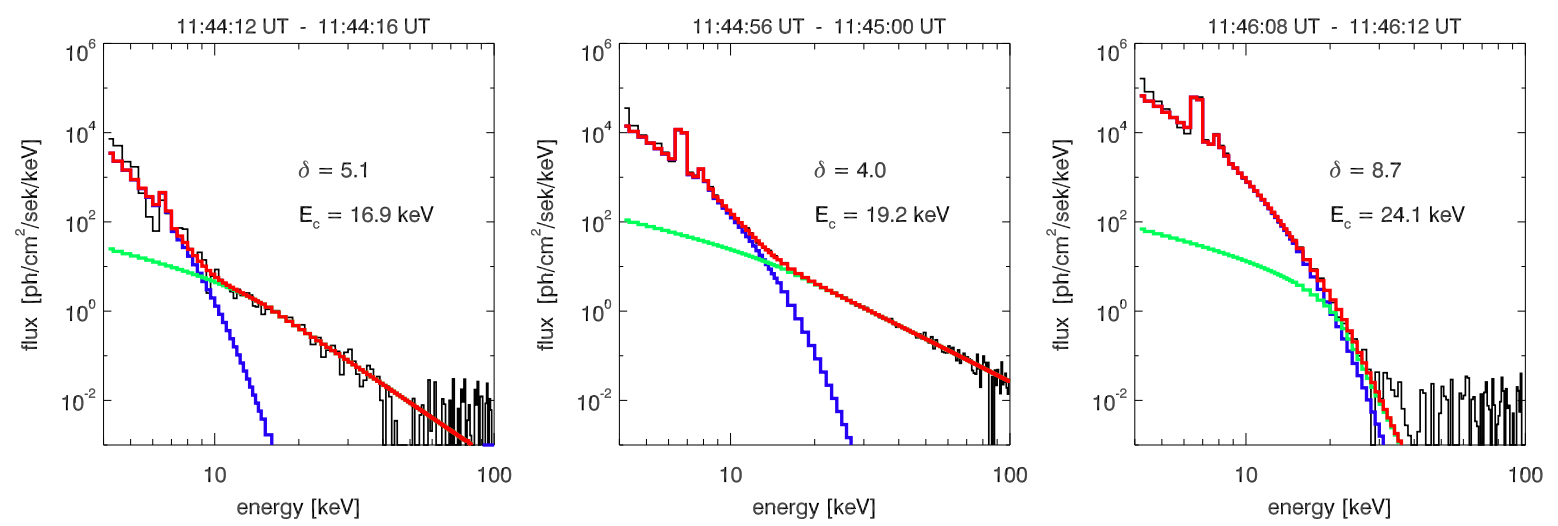

Fig. 6.- RHESSI spectra taken before (left panel), during (middle panel), and after the impulsive phase (right panel) of the flare on the June $2^{\text {nd }}, 2002$. The spectra were fitted with the single temperature thermal model (blue colour) and thick-target model (green). The total fitted spectra are shown in red. The obtained values $\delta$, and $\mathrm{E}_{\mathrm{c}}$ were determined on the basis of the thick-target model and used as characteristics of the injected electrons in the model.

(see a detailed description below). Furthermore, a comparative analysis of the spatial distributions of the signals in the RHESSI images and models of both flares was made. We compared signals emitted from selected structures and within selected energy ranges. The conformity level was applied as a measure of the model quality and validity of the applied procedures and simplifications.

\subsection{Modelling of the flares}

The RHESSI data were analysed using the RHESSI OSPEX package of the SolarSoftWare (SSW). The X-ray spectra of both flares were measured with a 4 seconds time resolution in 158 energy bands ranging from 6 to $300 \mathrm{keV}$. The analysed spectra were corrected for pulse pileup, decimation, and albedo effect (Compton back-scattering on the photosphere, Bai \& Ramaty (1978)) using the RHESSI standard analysis tools.

The accuracy of background subtraction is a key point in spectra analysis. In the case of the RHESSI spectra background is usually modelled as a linear (or higher-order polynomial) interpolation between averaged fluxes before and after the flare. However, for many flares and periods between them gradual rises and falls of this background were observed. Such a slow increase and then decrease of the flux were recorded for the February 20 $0^{\text {th }}, 2002$ flare before 11:04 UT. Similar background variations were observed in all energies, also in the highest energy channel where no flare emission was observed. Averaged time profiles of the high energy backgrounds were used for the modelling of the background variation in the low energy channel during this flare. This method allows for a subtraction of the backgrounds with sufficient accuracy in different energy channels. 
For the June $2^{\text {nd }}, 2002$ event the background varied marginally with time during this flare and was subtracted from data using a linear fit of the background fluxes recorded before and after the flare.

The RHESSI A1 attenuator was permanently used during both analysed events. Thus, counts registered in the 3-6 keV energy range are almost exclusively generated by higher energy photons above $11 \mathrm{keV}$, which is caused by the K-escape effect (Smith et al. 2002). Therefore, only counts above $6 \mathrm{keV}$ were analysed. For the February $20^{\text {th }}, 2002$ event above $90 \mathrm{keV}$ and for the June $2^{\text {nd }}$, 2002 above $100 \mathrm{keV}$ the X-ray background flux dominates over flare emission, so fluxes above these energy levels were also not included for spectral fitting.

Particularly at the beginning and end of the flare when recorded fluxes are low, the count rates in some energy bins can be negative as a result of background subtraction and low signal to noise ratios. In order to keep the count rates positive (particularly in the $6-20 \mathrm{keV}$ energy range) an increased accumulation time was applied, causing a worse time resolutions for the beginnings and ends of both analysed events than maxima phases. The spectra were fitted assuming single temperature thermal plus thick-target models. The thermal model was defined by a single temperature and an emission measure of the optically thin thermal plasma, based on the X-ray continuum and line emissions calculated using the CHIANTI atomic code (Dere et al. 1997; Landi et al. 2006). The thick-target model was defined by the total integrated non-thermal electrons flux $\mathrm{F}_{\mathrm{nth}}$, the powerlaw index of the electron energy distribution $\delta$, and low energy cut-off of the electron distribution $E_{c}$. These parameters were then used as characteristics of the injected electrons in the model. Figures 3 and 6 show examples of spectra and fits the thermal and non-thermal component for analysed events and determined on the basis of parameters of the thick-target model. The RHESSI spectra were fitted using a forward and backward automatic fitting procedure, starting from a time when the non-thermal component was strong enough and clearly visible. The obtained values of the fitted parameters were controlled and corrected if necessary. Generally, all fitted parameters evolved in a quasi-continuous manner.

Geometrical and physical models of the pre-flare loops were built using geometrical parameters - semi-length $\mathrm{L}_{0}$ and cross-section $\mathrm{S}$ and also thermodynamic parameters like initial pressure at the base of transition region $\mathrm{P}_{0}$, temperature, emission measure, mean electron density and GOES flux in the energy range 1-8 $\AA$ were estimated from RHESSI and GOES data. In order to obtain for initial pre-flare models the best conformity between theoretical and observed GOES light curves, the semi-lengths and cross-sections of the loops were refined in a range of \pm 1 pixel. Table 1 presents the values of $\mathrm{S}, \mathrm{P}_{0}$ and $\mathrm{L}_{0}$ used in the models of the analysed flares.

For each time step the momentary heating rate of plasma along the loop was estimated using Fisher's heating function. In this function thick-target parameters $\mathrm{F}_{\mathrm{nth}}, \delta$, and $\mathrm{E}_{\mathrm{c}}$ of the NTEs beams obtained from fitting consecutive RHESSI spectra were used. The time step of the NTEs beam was defined as the accumulation time needed to obtain the spectrum from RHESSI data 
Table 1: Cross-sections, semi-lengths and base pressure of the analysed flares.

\begin{tabular}{cccccccc}
\hline \hline $\begin{array}{c}\text { Event } \\
\text { date }\end{array}$ & \multicolumn{2}{c}{$\begin{array}{c}\text { Time of } \\
\text { start } \\
\text { maximum } \\
{[\mathrm{UT}]}\end{array}$} & $\begin{array}{c}\text { GOES } \\
\text { class }\end{array}$ & $\begin{array}{c}\text { Active } \\
\text { region }\end{array}$ & $\mathrm{S}$ & $L_{0}$ & $P_{0}$ \\
& & $\mathrm{AR}$ & {$\left[10^{16} \mathrm{~cm}^{2}\right]$} & {$\left[10^{8} \mathrm{~cm}\right]$} & {$\left[\right.$ dyn $\left./ \mathrm{cm}^{2}\right]$} \\
\hline 20-Feb-02 & $11: 02$ & $11: 07$ & $\mathrm{C} 7.5$ & 9825 & 7.16 & 17.2 & 63.0 \\
02-Jun-02 & $11: 41$ & $11: 47$ & $\mathrm{C} 9.4$ & 9973 & 5.81 & 8.37 & 72.0 \\
\hline
\end{tabular}

$\mathrm{S}$ and $\mathrm{L}_{0}$ - cross-section and semi-length of the flaring loop; $\mathrm{P}_{0}$ - pressure at base of transition region

with a sufficient $\mathrm{S} / \mathrm{N}$ ratio. For this reason, during each NTEs beam's time step a heating of the loop was calculated with fixed NTE beam parameters, while the thermodynamic parameters of the flaring plasma varied, being calculated with a much shorter time step of the HD model. The best beam time resolution was obtained when the flux was the highest around the maximum of event (being equal to 4 seconds), and the worst for the beginning and the end (a dozen or several dozen seconds). At the end of each NTE's beam time step GOES fluxes were calculated and compared with the observed GOES fluxes. If a conformity of the fluxes was achieved, the procedure was applied for the next time step. Otherwise, the $\mathrm{E}_{\mathrm{c}}$ value was carefully adjusted in order to achieve conformity of the observed and modelled GOES fluxes in the 1-8 $\AA$ energy band. A variation in the $E_{c}$ value of just a few $\mathrm{keV}$ can add or remove a substantial amount of energy to or from the flare loop because of the power-law nature of the energy distribution. Thus $\mathrm{E}_{\mathrm{c}}$ must be selected with high precision. Usually the value of $\mathrm{E}_{\mathrm{c}}$ obtained from fitting RHESSI spectra is fraught with a large error. However the applied method limits the range of $E_{c}$ using an independent energetic condition, like the observed 1-8 A GOES flux.

\subsection{Calculation of thermal emission}

The thermal emission fluxes were calculated using the CHIANTI atomic code (Dere et al. 1997; Landi et al. 2006) for the temperature range 1-50 MK, ionization equilibrium by Mazzotta et al. (1998) and solar coronal extended abundances. Thermal emission was calculated for both the lines and continuum. The procedure for calculating the thermal emission fluxes consisted of several stages. In the first, thermal emission was calculated for each cell of the model and in four energy ranges: $6-12 \mathrm{keV}, 12-25 \mathrm{keV}, 25-50 \mathrm{keV}$ and $50-100 \mathrm{keV}$ at the end of each NTE's beam time step. Next, the total flux was calculated for the entire loop in the same energy ranges. As a result the light curves in different energy ranges and spatial distribution of the thermal emission were obtained. 


\subsection{Calculation of non-thermal electron distribution}

In this work, the Focker-Planck formalism was applied using a code originally developed by Leach \& Petrosian (1981), based upon a program developed by Walt, MacDonald, and Francis (described in Carovillano \& McClay 1968), extended to ultrarelativistic energies by McTiernan \& Petrosian (1990) and prepared for public distribution by Holman (http://hesperia.gsfc.nasa.gov/hessi/ flarecode/efluxprog.zip). This code computes the steady-state electron distribution function in the flare's magnetic loop as a function of a position along a magnetic field line, electron pitch angle, and electron energy. Input data consist of injected flux, distribution of non-thermal electrons at the top of the loop, a model of a distribution of plasma in the flare's loop and a model of the magnetic field. The solution includes effects due to Coulomb collisions with the ambient plasma, effects due to synchrotron emission by the electrons, and effects of bremsstrahlung emission. It should be noted that most of the non-thermal electrons lose their energy in Coulomb collisions while a small fraction $\left(\sim 10^{-5}\right)$ of the electrons' energy is converted into HXR by the bremsstrahlung process. In addition, a synchrotron effect is insignificant in solar flares. An assumption of a steady-state imposes that the injected flux of NTEs is constant in time for all time scales smaller than the transport time scales. In other words, the time of flight of the NTEs from top of the loop to bottoms (feet) must be shorter than the interval, when parameters of the injected NTEs beam at the top of the loop varied. The code has been modified to work with hydrodynamic code in order to retrieve physical parameters of each cell along a computational loop. As a result the procedure calculates the electron flux distribution $\mathrm{F}(\mathrm{E}, \mathrm{s}, \mu)$ as a function of energy $\mathrm{E}$, at all points in the loop (distance from the top of loop s) and for all angles (pitch angles $\mu=\operatorname{cosine} \theta$ ) in electrons $/ \mathrm{cm}^{2} / \mathrm{sr} / \mathrm{sec} / \mathrm{keV}$. For both analysed flares, constant cross-section of loops was assumed (for what seems to be a good approximation, see Peter \& Bingert 2012) which implies no magnetic mirroring because magnetic field strength was constant and equal to 200 Gauss. The value has been selected on the basis

of estimates of the coronal magnetic field presented in Schmeltz et al. (1994) and Aschwanden (2005).

\subsection{Calculation of non-thermal emission}

Known distribution of NTEs along the loop allows for the calculation of the thin-target nonthermal bremsstrahlung radiation intensity $I(\varepsilon, s)$ at the end of each time step. The intensity is a function of photon energy $\varepsilon$ and distance from the top of loop s, and by using the formula of Tandberg-Hanssen \& Emslie (1988) one has:

$$
I(\varepsilon, s)=S \int_{\varepsilon}^{\infty} n_{p}(s) \sigma_{B} F(E, s) d E
$$


where $\mathrm{S}$ is a cross-section area of a loop, $\mathrm{n}_{\mathrm{p}}$ is the ambient proton density as a function of the distance along the injected electron's path for full ionization. $\sigma_{\mathrm{B}}$ is the angle-averaged differential bremsstrahlung cross-section given by Koch \& Motz (1959). Electron flux distribution $F(E, s)=\int_{-1}^{1} F(E, s, \mu) d \mu$ was integrated over the pitch angle from 1 to -1 . The $I(\varepsilon, s)$ is calculated in $\mathrm{ph} / \mathrm{cm}^{2} / \mathrm{sec} / \mathrm{keV}$. As a result the light curves and spatial distribution in different energy ranges of the non-thermal HXR emission is obtained.

\subsection{Observed and synthesized light curves}

Observed hard X-ray light curves in $\mathrm{ph} / \mathrm{cm}^{2} / \mathrm{sec} / \mathrm{keV}$ were restored on several assumptions: the RHESSI attenuator state during the analysed flares was permanently A1, the resulting fluxes were obtained by dividing the instrument response matrix with diagonal coefficients only, thus other factors were not taken into account e.g. there are no corrections for effects such as K-escape and Compton scattering (described by the off-diagonal elements of the detector response matrix). Both analysed flares were in the upper range of GOES class C (C7.5 and C9.4), which does not emit high hard X-ray fluxes. The correction of decimation and pileup was also included. Observed light curves were prepared in four energy bands: $6-12 \mathrm{keV}, 12-25 \mathrm{keV}, 25-50 \mathrm{keV}$ and $50-100 \mathrm{keV}$. To make a comparison of modelled and observed fluxes possible, it was necessary to add to each of them the HXR thermal and non-thermal synthesized component and then add a background set of observational light curves. In this way it was possible to compare the synthesized and observed fluxes in the same energy range selected. Figure 7 shows a schematic flow diagram used for the modelling of solar flares and their analysis. All of the components in the diagram are described in this section.

\section{Results}

The main results of modelling the analysed flares are presented in Figures 8 and 9 for June $2^{\text {nd }}, 2002$ and Figures 10 and 11 for Feb 20 ${ }^{\text {th }}, 2002$. The synthesized GOES 1-8 $\AA$ light curves of the flaring loops follow closely those observed for the analysed flares, which is a direct result from our assumptions. The real test for the accuracy of the model is to compare the observed and calculated fluxes in the 0.5-4 $\AA$ band. The quality of the fit between an observed and synthesized light curve in the 0.5-4 $\AA$ band would be a good measure of quality of the calculated flare model. In other words, with smaller differences between light curves in the 0.5-4 $\AA$ band, one gets a better model/simulation, larger differences, and a worse model/simulation. For the event of June $2^{\text {nd }}$, 2002 one can trace this effect in Figure 8 (upper left panel). The calculated light curves for the analysed event did not differ too much from the observed ones. It seems that our model simulates 


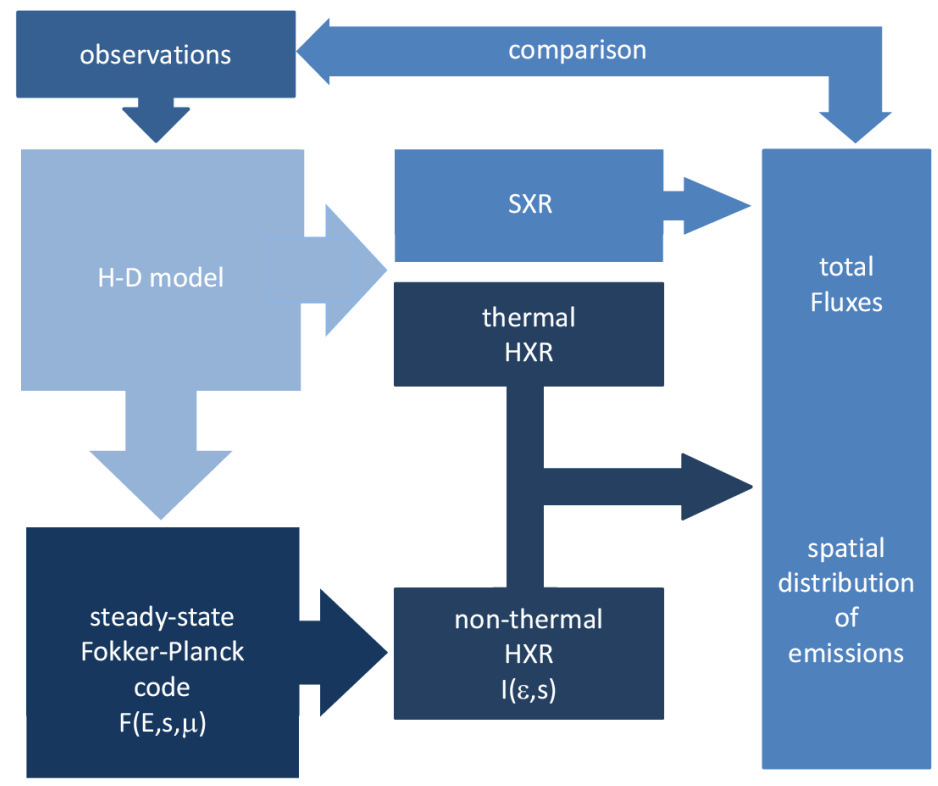

Fig. 7.- Flow diagram showing the procedures used during modelling and analysis of flares.

the main physical processes in the right way despite the large simplifications which are used. At the beginning of the flare the synthesized light curve (red colour) is under observed (blue dashed line), after 11:44:20 UT have a similar pattern to the maximum at 11:45:40 UT. After this time, during the flat maximum the synthesized curve is located again under the observed one to 11:48:20 UT. Again, after this time the curves are in a similar course to the end of the simulation. Additionally, one can see that during the decay phase (after 11:48:20 UT) some heating is necessary. The green dotted curve in Figure 8 (upper left panel) represents the evolution of the plasma in the flaring loop without any heating and has a different course to the observed one. The observation corresponds well to the evolution of plasma flares in the loop which is heated in this time. A difference in the course between synthesized and observed light curves also reflects on the calculated temperature and emission measure values. We can follow the behaviour temperature with time in Figure 8 (upper right panel). Obviously, where there are the greatest differences in the flux 0.5-4 $\AA$ energy band, there will also be the largest differences between the temperatures determined from observations and synthesized curves. From almost the beginning of the flare, the temperature is lower than that determined at the same time from observations but both values agree within the range of errors. The situation changes after 11:44:20 UT when the temperature calculated from the model is higher than that calculated from observations and is outside the range of errors. About 11:45 UT there is a short episode when the temperatures determined from the model and observations are almost identical. After 11:45:20 UT the highest difference between the temperatures calculated from observations and from the model is observed. At about 11:46:20 UT the difference is at its greatest and is about $3 \mathrm{MK}$. After this time the difference gradually decreases to reach a similar value at 

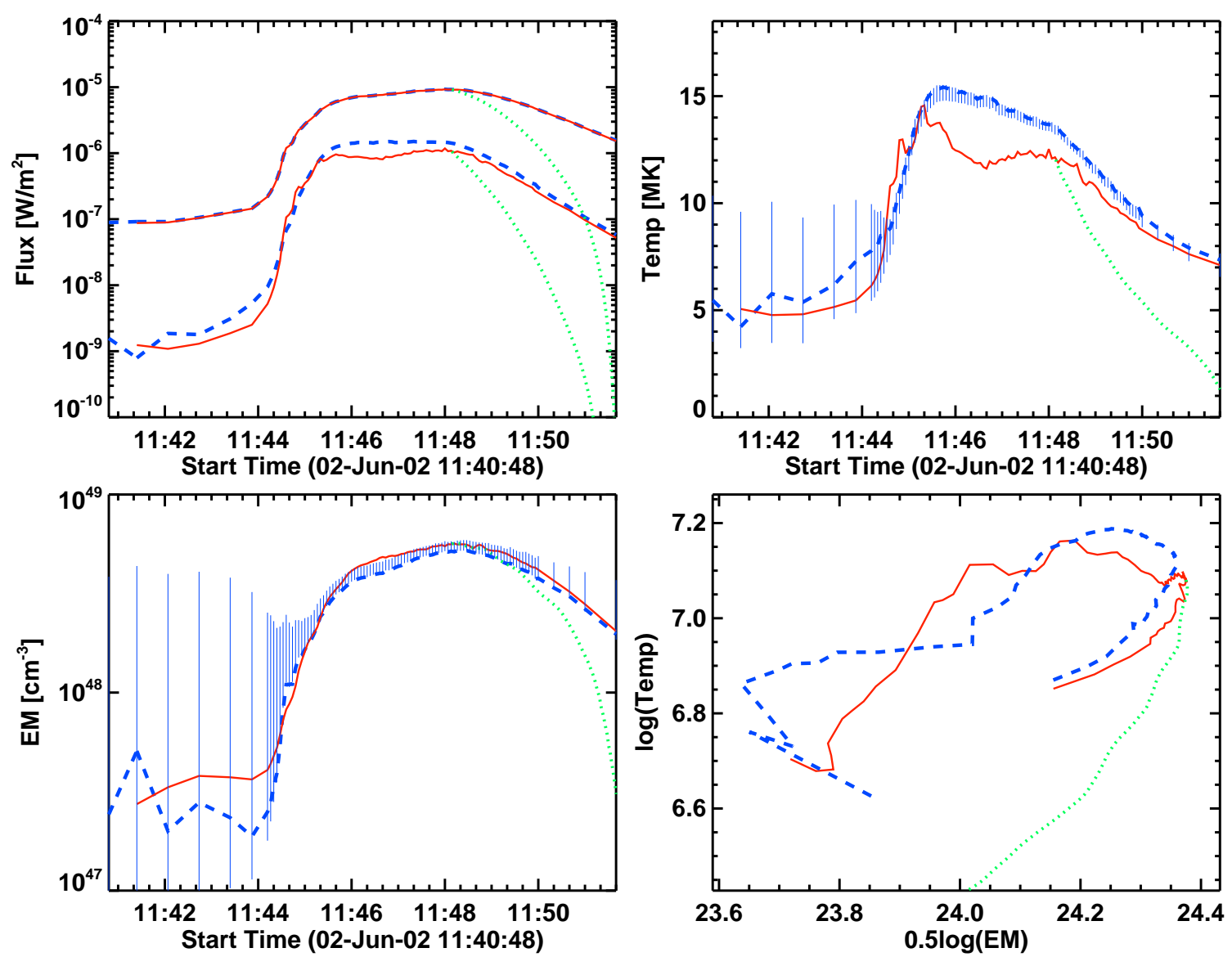

Fig. 8.- Results of the modelling of the June $2^{\text {nd }}, 2002$ solar flare. The upper left panel presents the observed and calculated GOES fluxes in 0.5-4 $\AA$ (lower curve) and 1-8 A (upper curve) energy bands. The upper right panel shows temperature, lower left panel emission measure, and the lower right panel presents the diagnostic diagram $-\log (\mathrm{T})$ vs. $0.5 \log (\mathrm{EM})$. Colour code as used in the described panels in Figure: blue dashed lines relate to the value observed or calculated from GOES fluxes, red lines show the value calculated from the solar flare model, green (dotted) lines describe the value calculated from the solar flare model without any heating after 11:48:08 UT.

11:51:20 UT (again within the range of errors). The behaviour of the emission measure over time can be seen in Figure 8 (lower left panel). Generally, it can be said that apart from the beginning, an emissions measure calculated from synthesized light curves reproduces the course of emission measure calculated from observations very well, and generally their ranges of errors overlap apart from a few discrepancies. A very useful tool to observe the thermal evolution of the flaring plasma is a diagnostic diagram. This type of flare thermal analysis was presented for the first time by Jakimiec et al. (1992). On the diagnostic diagram the emission measure $(0.5 \log \mathrm{EM})$ is given on the horizontal axis while plasma temperature $(\log \mathrm{T})$ is given on the vertical axis. Generally, the 
evolutionary paths in the diagrams are very sensitive to the efficiency of the plasma heating during the flare. This is particularly important and relevant to the cooling phase of the flare when the energy flux is reflected by the slope of its evolutionary path. During this phase evolution of the flare can occur in two characteristic limiting branches, firstly, the branch when heating of the flaring loop is abruptly switched off and in this case, the slope of the evolutionary branch is about 2 . This is an instance of free or nearly-free evolution of the flaring loop, when heating decreases so quickly that it does not affect the rate of flare cooling. Secondly, the quasi-stationary state (QSS) branch

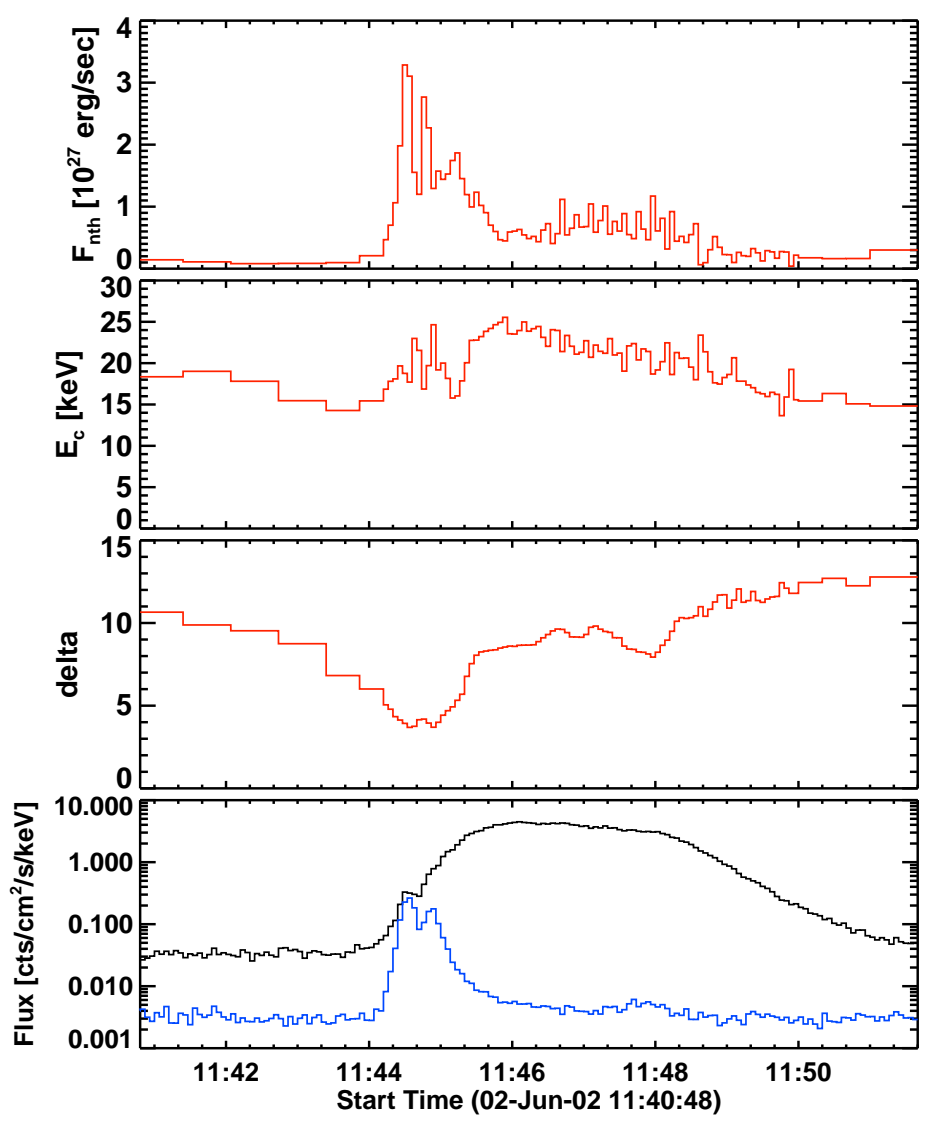

Fig. 9. - Time evolution of the thick-target model parameters calculated for the June $2^{\text {nd }}, 2002$ solar flare using RHESSI registered fluxes. From top to bottom: energy flux of non-thermal electrons $\mathrm{F}_{\text {nth }}$, low cut-off energy $\mathrm{E}_{\mathrm{c}}, \delta$ index of energy spectrum, and HXR fluxes: 12-25 keV (black line) and $25-50 \mathrm{keV}$ (blue line).

when the rate of flare cooling is still fully covered by the heating and the slope of the evolutionary path in this case is about 0.5 . Figure 8 (lower right panel) shows the diagnostic diagram for the June $2^{\text {nd }}, 2002$ solar flare. The difference between the observed and modelled evolutionary paths is caused mainly by underestimation of the modelled temperatures of plasma. During the cooling phase of the flare of June $2^{\text {nd }}, 2002$ from 11:48:20 UT to 11:50:20 UT the observed and modelled evolutionary paths have slopes which are very close to, but less than 2, after the time 11:50:20 UT 
the slope was about 0.5 . This behaviour indicates that some heating was present during the decay phase of the analysed event. A green dotted curve shows the evolutionary path calculated in the case when heating of the flaring loop was abruptly switched off after 11:48:08 UT, and the path reaches a slope very close to 2 after 11:48:50 UT.
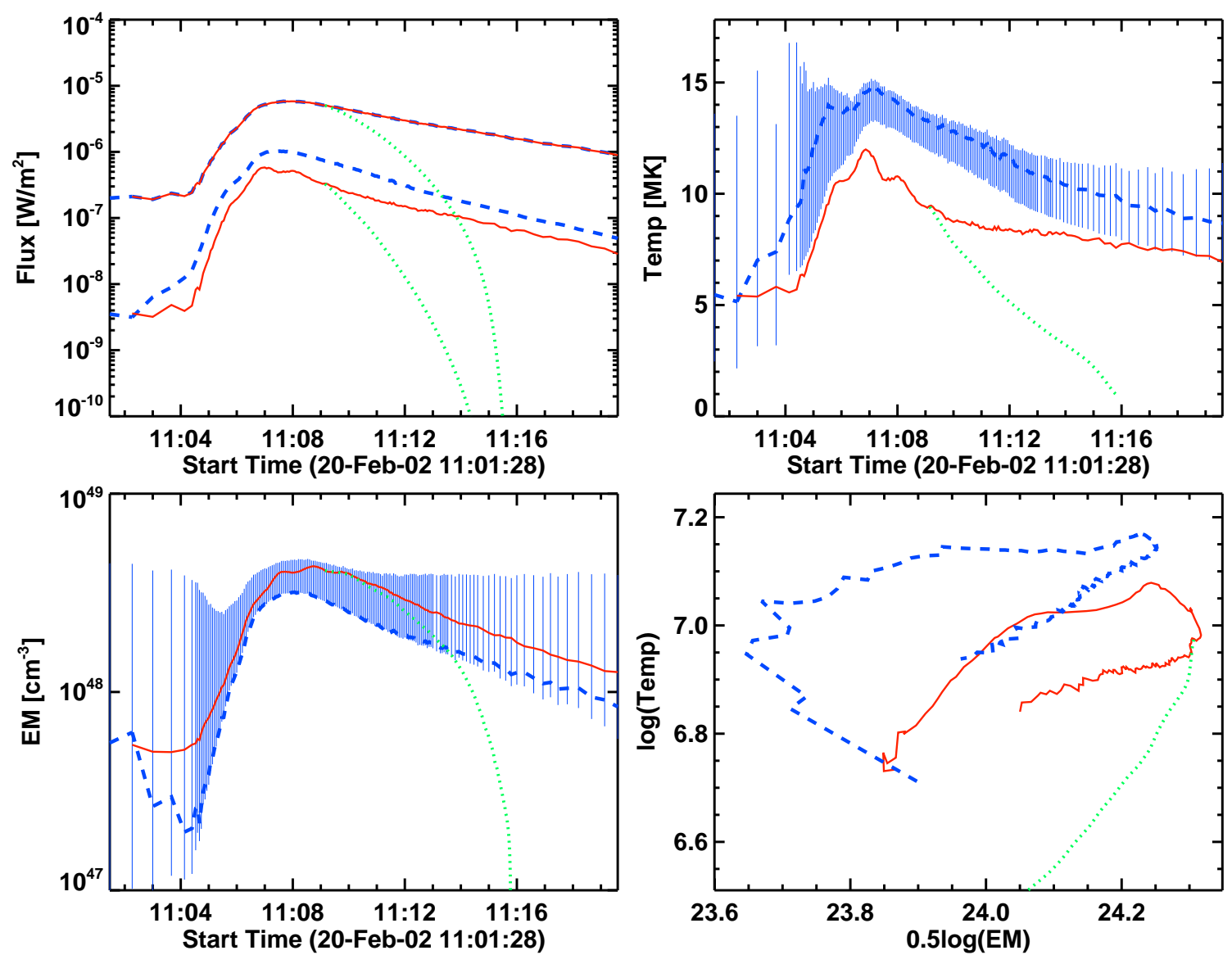

Fig. 10.- Results of the modelling of the February $20^{\text {th }}, 2002$ solar flare. The upper left panel presents the observed and calculated GOES fluxes in 0.5-4 $\AA$ (lower curve) and 1-8 $\AA$ (upper curve) energy bands. The upper right panel shows temperature, lower left panel emission measure, and lower right panel presents diagnostic diagram $-\log (\mathrm{T})$ vs. $0.5 \log (\mathrm{EM})$. Colour code as used in the described Figure: blue dashed lines relate to the value observed or calculated from GOES fluxes, red lines show the value calculated from the solar flare model, green (dotted) lines describe the value calculated from the solar flare model without any heating after 11:08:36 UT.

Figure 9 presents time variations of the electron beam parameters determined assuming thick target model and RHESSI fluxes in two energy bands, $12-25 \mathrm{keV}$ and $25-50 \mathrm{keV}$, respectively. As assuming a already mentioned earlier, non-thermal electron beams characterized by these parameters provide to the loop energy sufficient to obtain compliance of the observed and modelled GOES 
fluxes in a 1-8 $\AA$ energy band before and during the impulsive phase, and also during the decay phase of the flare. The time course of energy flux of NTE $F_{n t h}$ varied between $4.0 \times 10^{25}$ and $3.28 \times 10^{27} \mathrm{erg} \mathrm{s}^{-1}$, reaching the first maximum at 11:44:40 UT, and the second (strongly blurred) at 11:47:42 UT. These two maxima are reflected well in the course of the electron spectral index $\delta$, as local minima, when spectrum of non-thermal electrons was hardened. This behaviour is called in the literature as soft-hard-soft and most of the solar flares show during the impulsive phase this pattern of variation of the observed spectral index (see e.g., Grigis \& Benz 2004). These two maxima are also faithfully reproduced in the light curves recorded by RHESSI, especially in the 25-50 $\mathrm{keV}$ energy range where the first maximum (11:44:40 UT) reflects the main impulsive phase of the flare and a second maximum seen as a small increase in the flux of 25-50 keV responsible for flattening the SXR maximum observed at the same time for GOES emission and RHESSI channels 4-12 keV and 12-25 keV at 11:47:42 UT. Low energy cut-off $E_{c}$ varied during the flare between $14 \mathrm{keV}$ and $25 \mathrm{keV}$. During the modelled pre-heating phase of the flare $\mathrm{E}_{\mathrm{c}}$ value decreases from 18 $\mathrm{keV}$ to $14 \mathrm{keV}$, then after several sharp changes increases up to $25 \mathrm{keV}$ during the impulsive phase, and decreases back to $15 \mathrm{keV}$ during the gradual phase of the flare. Temporal variations of the calculated cut-off energy $E_{c}$ agree well with estimations of $E_{c}$ range and temporal variations made in a previous paper (Falewicz, Siarkowski, \& Rudawy 2011) and already presented by various authors, e.g., Holman et al. (2003); Sui et al. (2007) and Warmuth et al. (2009a.b).

Figure 10 presents the results of modelling of the February $20^{\text {th }}, 2002$ solar flare. In the case of this event quality of the restoration of GOES 0.5 - $4 \AA$ light curve (upper left panel) is much worse compared to the June $2^{\text {nd }}, 2002$ solar flare. For almost the entire duration of the analysed flare the modelled light curve $(0.5-4 \AA)$ is located below the observed light curve but an overall pattern of the variations is very similar to the observed ones. The courses of the light curves show, that during the decay phase some heating occurred. The green dotted curves in Figure 10 (upper left panel) represent the evolution of the plasma in the flaring loop without any heating after 11:08:36 UT and have different courses than the observed ones. A differences in the courses between synthesized and observed light curves, as in the case of the first analysed event, also reflects on the calculated temperature and emission measure values. For temperatures (upper right panel) the biggest difference is observed for the maximum of the flare, at 11:07:10 UT, when the difference between the temperatures determined from observations and synthesized light curves reached about $3 \mathrm{MK}$. After the maximum of the flare the difference between temperatures gradually decreases to reach a value of about 1.5 MK at 11:19:40 UT. Temperatures determined from a synthesized light curves only at the beginning and end of the event are within the range of errors of temperatures calculated from the observed light curves. Time variations of emission measure are presented in Figure 10 (lower left panel). Emission measure calculated from the synthesized light curves reproduce the course of emission measure calculated from observation but observed EMs are higher. For the whole event the course of EM calculated from a synthesized light curve 


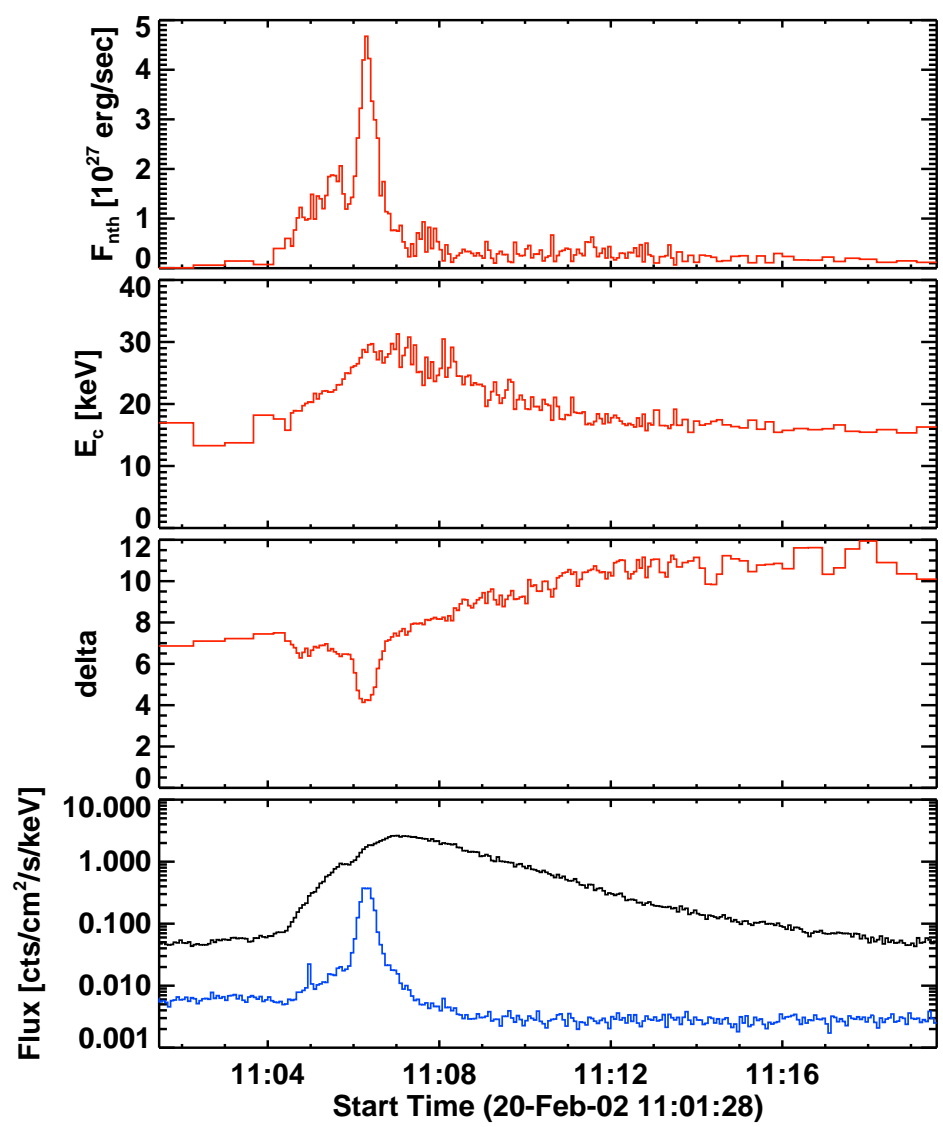

Fig. 11. - Time evolution of the thick-target model parameters calculated for the February $20^{\text {th }}$, 2002 solar flare using RHESSI fluxes. From top to bottom: energy flux of non-thermal electrons $\mathrm{F}_{\text {nth }}$, low cut-off energy $\mathrm{E}_{\mathrm{c}}, \delta$ index of energy spectrum, and HXR fluxes: $12-25 \mathrm{keV}$ (black line) and $25-50 \mathrm{keV}$ (blue line).

is within the range of errors of EM calculated from the observed light curves. The main difference between observed and modelled evolutionary paths on the diagnostic diagram (Figure 10, lower right panel) is caused by underestimation of temperature and overestimation of emission measure. However, despite the shift both evolutionary paths are similar. During the cooling phase of the flare from 11:16:20 UT to 11:19:30 UT the observed and modelled evolutionary paths have a similar slope of about 0.5 and this proves that some heating was present during the decay phase. The time variation of the parameters of the electron beam in thick target approximation and RHESSI fluxes of the flare are presented in Figure 11. The energy flux of NTEs $F_{n t h}$ varied in time between $5.7 \times 10^{24}$ and $4.7 \times 10^{27} \mathrm{erg}^{-1}$ reaching a maximum at 11:06:20 UT. Low energy cut-off $\mathrm{E}_{\mathrm{c}}$ varied during the analyzing flare between $13 \mathrm{keV}$ and $31 \mathrm{keV}$ and has a similar pattern of variations to the previously analysed event. Electron spectral index $\delta$ varied between 4 and 12, exhibiting typical behaviour of variation soft-hard-soft observed during the impulsive phase for most solar flares. The observed minimum for the electron spectral index is reflected in the maximum 
of the light curve recorded by RHESSI in the $25-50 \mathrm{keV}$ energy range at 11:06:08 UT. Previously (Siarkowski, Falewicz, \& Rudawy 2009; Falewicz, Siarkowski, \& Rudawy 2011), and in the present work, optimisation of the low energy cut-off of the electron distribution $\mathrm{E}_{\mathrm{c}}$ by comparison of the observed and calculated 1-8 $\AA$ GOES fluxes is used during the modelling of flares. However, the conformity of the observed and synthesized light curves in the $0.5-4 \AA$ band serves as a measure of quality of the calculated flare models. In this approach, these conditions are treated as independent in energy, but it is worth noting that the two energy ranges for GOES overlap. Therefore, it was decided to check in this work the accuracy of the model for other energy ranges. The method is based on a comparison of the synthesized and observed total fluxes in 6-12 keV, 12-25 keV, 25-50 $\mathrm{keV}$ and 50-100 keV energy bands. Examples of such comparisons are shown in Figures 12 and 13 respectively for the July $2^{\text {nd }}, 2002$ and February $20^{\text {th }}, 2002$ solar flares. For the solar flare of June $2^{\text {nd }}, 2002$ (Figure 12) the conformity of the observed and synthesized light curves in higher energy bands (25-50 keV and 50-100 keV) is very good. In these bands of energy the largest contribution to the total flux comes from a non-thermal emission. In lower energy ranges, the contribution of this component decreases and the thermal component begins to play a dominant role. Observed $\mathrm{X}$-ray light curves are of course the sum of the thermal and non-thermal emissions. Comparison of the observed light curves with calculated thermal and non-thermal fluxes, can shows what point in time what type of emission dominated during the various phases of the flare. In Figure 12 the non-thermal synthesised emission in the energy range $50-100 \mathrm{keV}$ is represented by the dark blue (dotted) curve. For the lower range $(25-50 \mathrm{keV})$ synthesized emission is represented by the red curves, which are sums of synthesized thermal and non-thermal emissions, but the contribution of thermal emissions in this energy range is still very small. It is only in the energy range of 12-25 $\mathrm{keV}$ that thermal and non-thermal emissions have comparable contributions. For example, for this range of energy temporary dominance of one of the components can be traced. After 11:44:00 UT the non-thermal component dominates, which is associated with the main impulsive phase of the flare, and at 11:45:10 UT thermal emission begins to dominate up to 11:50:00 UT, where again the two components have similar contributions. The quality of the fit between an observed and synthesized light curve for the $12-25 \mathrm{keV}$ energy band is worse than at higher energy ranges. In particular, the differences are apparent after 11:44:20 UT, where the maximum of the synthesized light curve is lower compared to the maximum of the observed light curve. The shape of the maximum of the synthesized light curve is restored correctly and can be seen in the double peaks as in observed light curves.

After 11:45:10 UT a local minimum appears in the synthesized light curve which does not occur for the observed light curve. A similar effect at the same time, appeared for the observed light curve of GOES 0.5-4 $\AA$ band (see Figure 8, upper left panel). The non-thermal component does not indicate the described behaviour, therefore this effect corresponds to the thermal component. Again, after 11:48:30 UT the curves are in a similar course to the end of the simulation. 


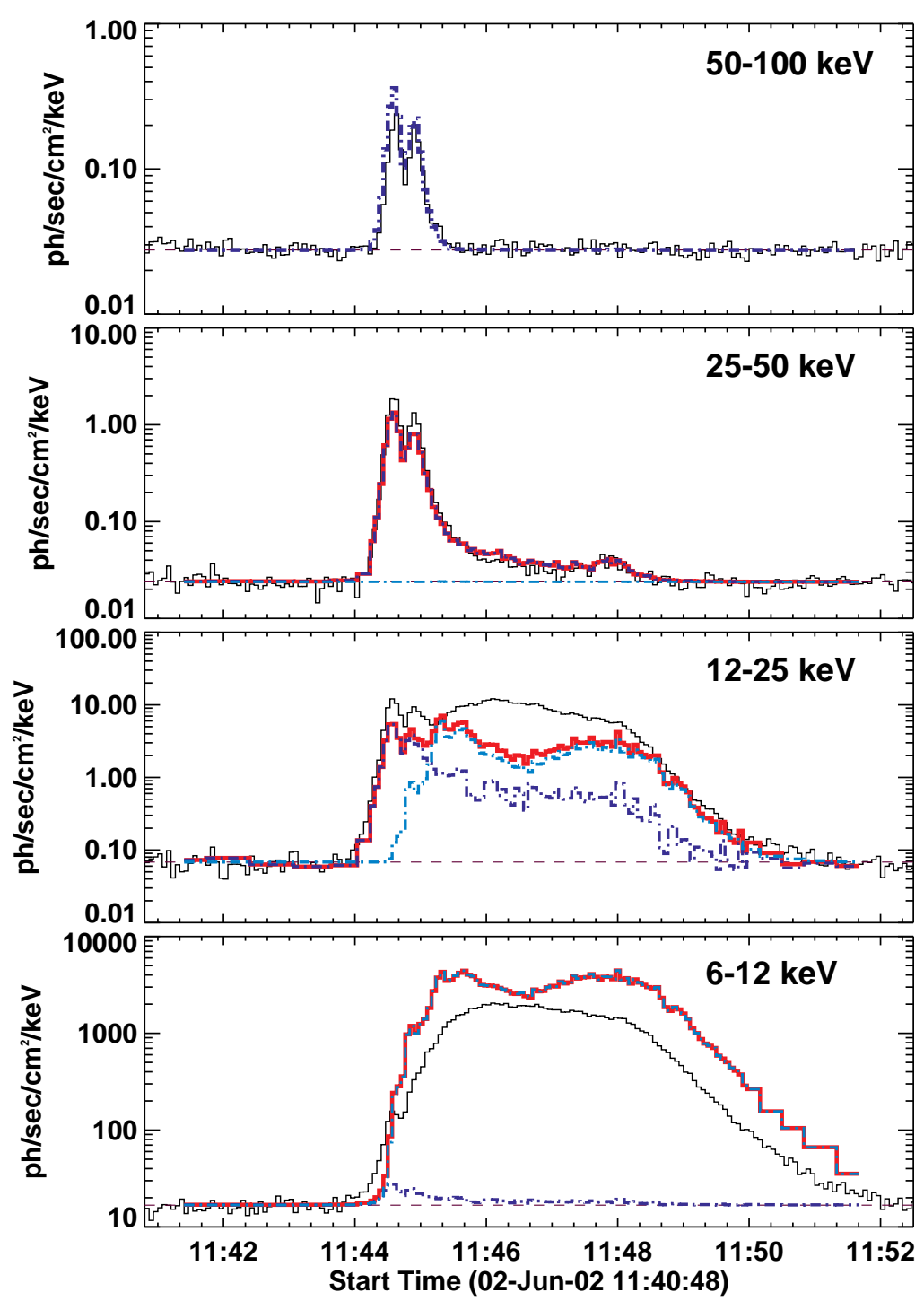

Fig. 12.- Results of the modelling of the June $2^{\text {nd }}, 2002$ solar flare. The figure shows synthesized and observed total fluxes in 50-100 keV, 20-25 keV, 12-25 keV and 6-12 keV energy bands. Colour code as used in the described Figure: the black curves - observation light curves from RHESSI. The dark blue (dotted) curves - synthesized non-thermal emission. The bright blue (dotted) curves - synthesized thermal emission. The red curves - sum of synthesized thermal and non-thermal emissions. The dashed brown line is a background derived from observations.

In the lowest energy channel $(6-12 \mathrm{keV})$ the thermal component dominates, although during the impulsive phase the non-thermal component gives a small contribution. The synthesized emission has a similar pattern of the variation, but runs over the observed light curve. Also in this energy band a local minimum of the synthesised light curve appears at 11:46:35 UT which does not occur 


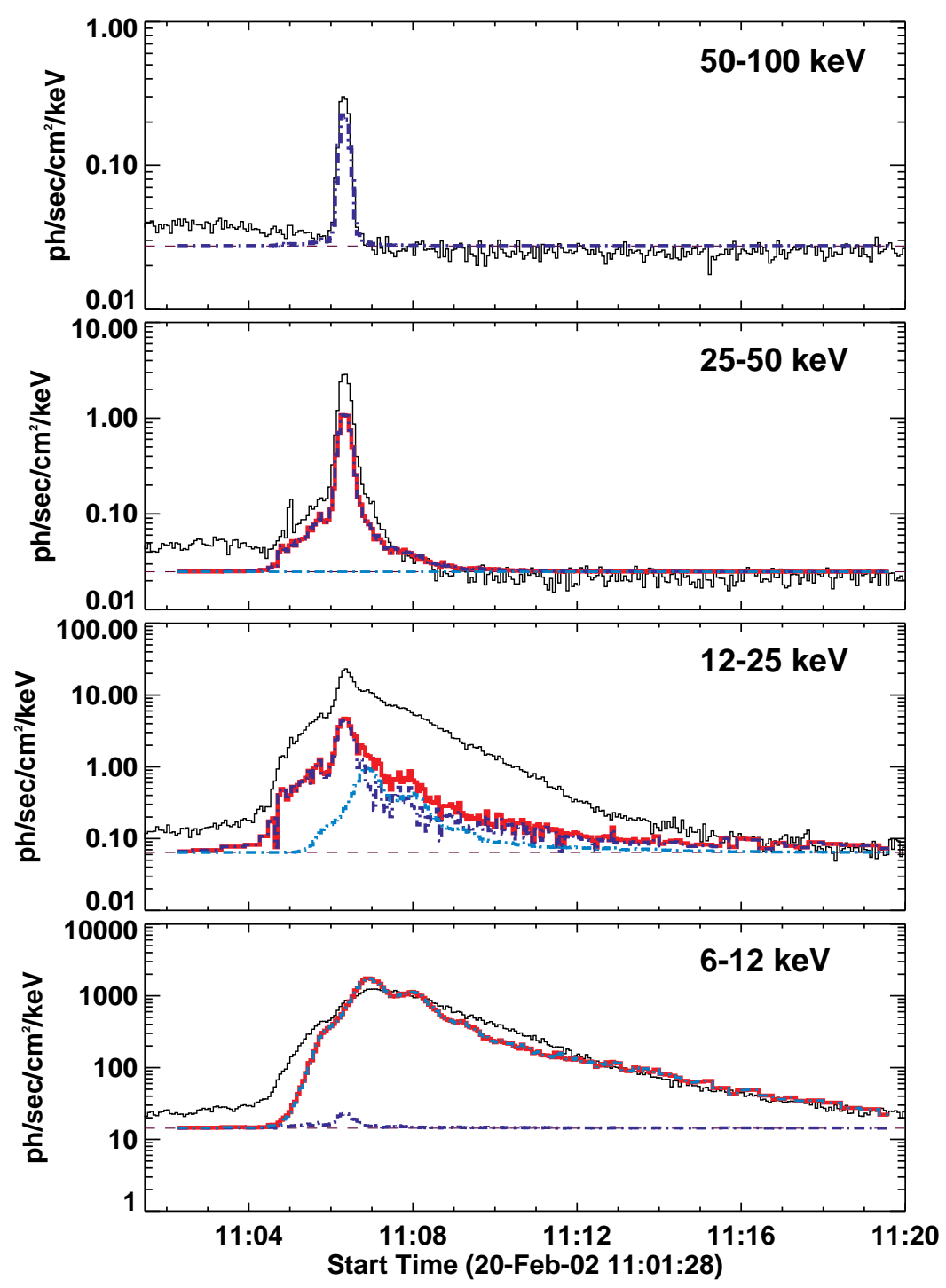

Fig. 13. - Results of the modelling of the February $20^{\text {th }}, 2002$ solar flare. The figure shows the synthesized and observed total fluxes in 50-100 keV, 20-25 keV, 12-25 keV and 6-12 keV energy bands. The black curves - observation light curves from RHESSI. The dark blue (dotted) curves synthesized non-thermal emission. The bright blue (dotted) curves - synthesized thermal emission. The red curves - sum of synthesized thermal and non-thermal emissions. The dashed brown line is a background derived from observations.

in observed light curve. The emission calculated from the model in the 6-12 keV begins to clearly start and grows later and faster than observed one, but this behaviour is not observed in the other described energy bands.

Figure 13 shows the comparison of the synthesized and observed total fluxes in the same four 


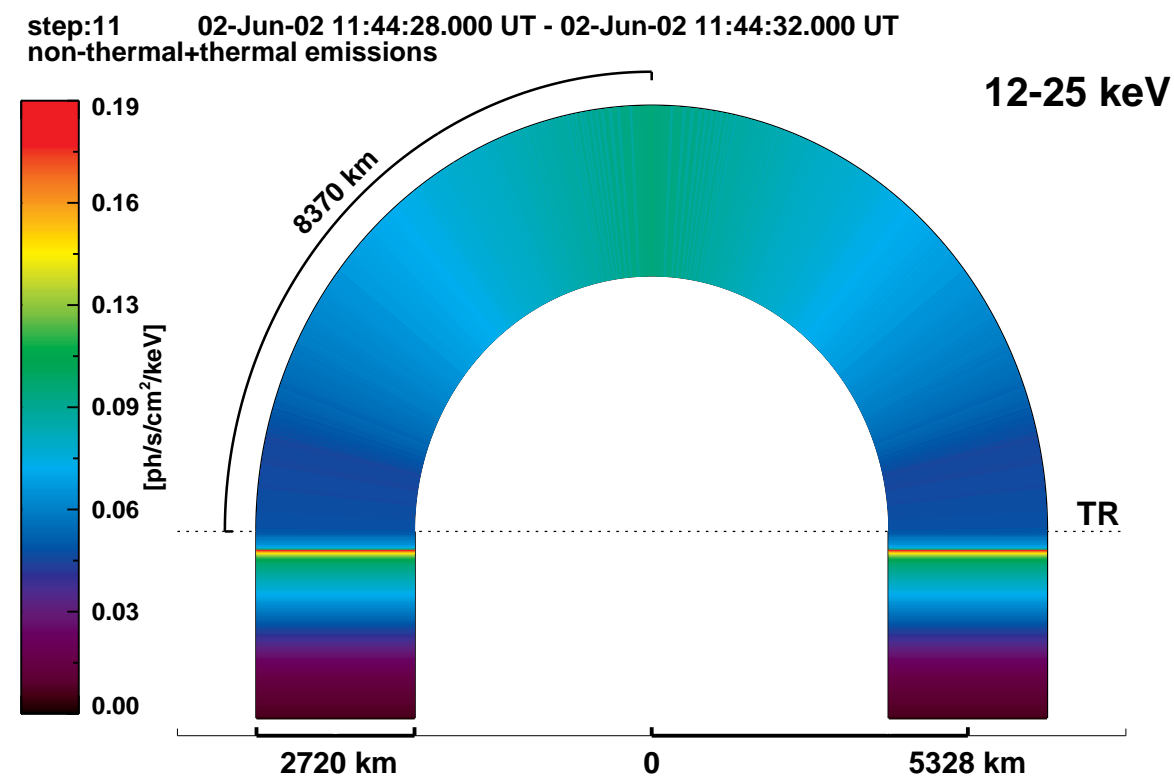

Fig. 14. - An example of the simulation of the spatial distribution of integral non-thermal and thermal emission in the energy range $12-25 \mathrm{keV}$ calculated for a selected evolution stage of the June $2^{\text {nd }}, 2002$ flare. The dashed line represents a level of the transition region (TR). Impulsive brightenings in the feet of the loop are well seen while the thermal emission at this time does not contribute (more details in the main text).

energy bands of the February $20^{\text {th }}, 2002$ solar flare. For this event quality of the restoration of synthesized curves is worse in relation to the June $2^{\text {nd }}, 2002$ solar flare similarly as for GOES 0.5-4 $\AA$ light curve. For almost the entire duration of the analysed flare the modelled light curves are located below the observed light curves, but the variation patterns are very similar to the observed ones. Only in the 6-12 keV energy band the synthesized curve has a similar course and values to the observed light curve, but at the beginning, the start of emission calculated from the model begins clearly later than in observation, just as in the case of the first analysed flare. The biggest differences between synthesized and observed light curves are in the energy range of 12-25 keV and they decrease with increasing energy.

Figure 14 presents an example of the simulation of the spatial distribution of integral nonthermal and thermal emission in the energy range $12-25 \mathrm{keV}$, calculated for the early-stage of the impulsive phase of the June $2^{\text {nd }}, 2002$ flare. The emission comes mainly from the feet, where impulsive brightenings appeared caused by non-thermal electrons which heat chromospheric matter. A much weaker and more diffuse emission comes from the top of the loop, which also has a nonthermal origin. A more complex picture of the flare is shown in Figure 15. This is an example of the simulation of spatial distribution of total non-thermal and thermal component calculated also for the early-stage of the impulsive phase of the February $20^{\text {th }}, 2002$ flare. In this case, as can be 


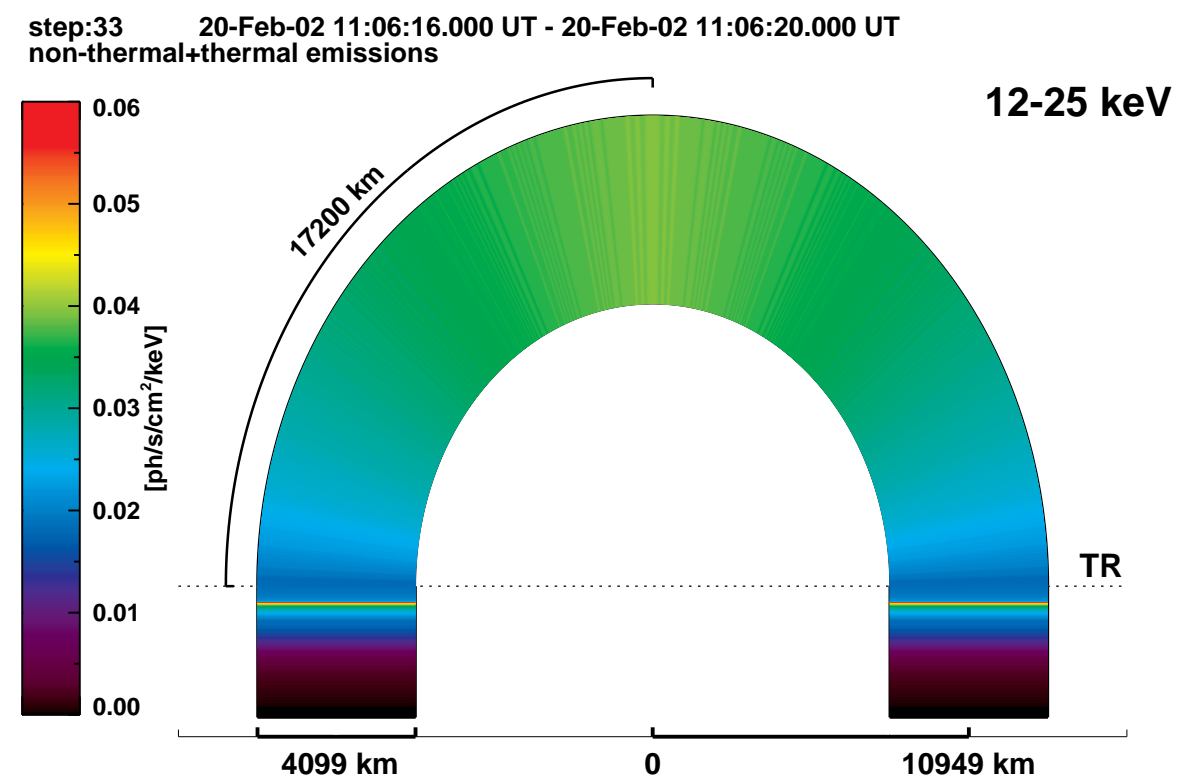

Fig. 15. - Simulation of the spatial distribution of total non-thermal and thermal emission in the energy range 12-25 keV, calculated for a selected evolution stage of the February 20 ${ }^{\text {th }}$, 2002 flare. The dashed line represents the level of transition region (TR). Impulsive brightenings in the feet and a bright top of the loop are well seen (more details in the main text).

seen in the feet there is strong impulsive brightening and also much weaker and blurry emission, which is mainly thermal in origin, from the top of the loop.

Finally, spatial distributions of the signal in the RHESSI images were compared with emissions resulting from the model. Using the spatial distribution of the observed signals presented in Figures 2 and 5 (left panels) fluxes were measured for the selected structures and compared with the values obtained from the models for both analysed flares. The results are presented in Table 2 . For selected energy ranges $35-45 \mathrm{keV}$ and 30-80 keV emissions have a non-thermal origin, which can be proven by looking at the total fluxes in Figures 12 and 13 for June 2 ${ }^{\text {nd }}, 2002$ and February $20^{\text {th }}, 2002$, respectively. On the RHESSI images emissions originate chiefly from the feet of the flaring loops. For this reason, in the images were selected areas of the feet using the two connected methods. The first one delimits the interest area using the box defined as coordinates of the corners (see Table 2), the second delimits the feet with an isophote of $30 \%$ intensity of the brightest pixel in this box. In this way the areas of footpoints were estimated in the images of both analysed events. However, the observed fluxes of the footpoints are not identical (see Table $2-F P_{1}$ and $F P_{2}$ ). Therefore, taking into account the symmetry of the numerical model, the corresponding parameter was calculated as an average of the fluxes of both feet $\left(F P_{a v e}\right)$. The non-thermal footpoint flux is calculated for the model using formula (1) and additionally from $F_{F P}=\int_{\varepsilon_{1}}^{\varepsilon_{2}} \int_{s_{T R}}^{s_{M A X}} I(\varepsilon, s) d s d \varepsilon$ as total intensity below transition region $\left(\mathrm{s}_{\mathrm{TR}}\right)$ and integrated in selected energy ranges $(\varepsilon)$. For these 
Table 2: Comparison of observed and modelled fluxes for selected structures of the analysed flares.

\begin{tabular}{|c|c|c|c|c|c|}
\hline$\overline{\text { DATE }}$ & & February $20^{\text {th }}$, & $\overline{2002}$ & $\overline{\text { June } 2^{\text {nd }}, 2}$ & \\
\hline ACCUMULATION TIME & & 11:06:16 UT & $(8 \mathrm{~s})$ & 11:44:20 UT & $48 \mathrm{~s})$ \\
\hline & UNITS & OBSERVATIONS & MODEL & OBSERVATIONS & MODEL \\
\hline $\begin{array}{l}\text { ENERGY RANGE } \\
\text { ISOPHOTE } 30 \%\end{array}$ & & $35-45 \mathrm{keV}$ & & $30-80 \mathrm{ke}$ & \\
\hline$F P_{1}$ & $\mathrm{ph} / \mathrm{s} / \mathrm{cm}^{2} / \mathrm{keV}$ & $0.68-3.61$ & - & $2.37-6.61$ & - \\
\hline $\mathrm{FP}_{2}$ & $\mathrm{ph} / \mathrm{s} / \mathrm{cm}^{2} / \mathrm{keV}$ & $2.23-6.38$ & - & $1.09-4.41$ & - \\
\hline $\begin{array}{l}F P_{A V E} \\
\text { вох }\end{array}$ & $\mathrm{ph} / \mathrm{s} / \mathrm{cm}^{2} / \mathrm{keV}$ & $1.46-4.99$ & 0.81 & $1.73-5.51$ & 7.64 \\
\hline$F P_{1}$ & $\begin{array}{c}\mathrm{ph} / \mathrm{s} / \mathrm{cm}^{2} / \mathrm{keV} \\
\operatorname{arcsecs}\end{array}$ & 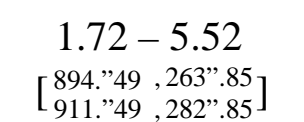 & - & $\begin{array}{c}3.93-9.02 \\
{\left[\begin{array}{c}-149^{\prime \prime} .40,-308^{\prime \prime}, 32 \\
-137^{\prime}, 40,-291.32\end{array}\right]}\end{array}$ & - \\
\hline$F P_{2}$ & $\begin{array}{c}\mathrm{ph} / \mathrm{s} / \mathrm{cm}^{2} / \mathrm{keV} \\
\operatorname{arcsecs}\end{array}$ & $\begin{array}{c}5.29-11.00 \\
{[891 \%, 49,262 \%, 85} \\
923 \%, 49\end{array}$ & $\begin{array}{l}- \\
-\end{array}$ & 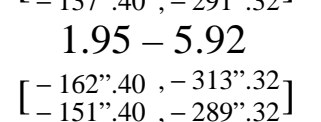 & $\begin{array}{l}- \\
-\end{array}$ \\
\hline$F P_{A V E}$ & $\mathrm{ph} / \mathrm{s} / \mathrm{cm}^{2} / \mathrm{keV}$ & $3.50-8.26$ & 0.81 & $2.94-7.47$ & 7.64 \\
\hline$F_{B K G}$ & $\begin{array}{c}\mathrm{ph} / \mathrm{s} / \mathrm{cm}^{2} / \mathrm{keV} \\
\operatorname{arcsecs}\end{array}$ & 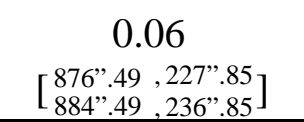 & $\begin{array}{l}- \\
-\end{array}$ & $\begin{array}{c}0.07 \\
{\left[\begin{array}{l}-177^{\prime \prime}, 40,-334^{\prime \prime}, 32 \\
-165^{\prime}, 40-325^{\prime \prime} .32\end{array}\right]}\end{array}$ & $\begin{array}{l}- \\
-\end{array}$ \\
\hline $\begin{array}{l}\text { ENERGY RANGE } \\
\text { ISOPHOTE } 30 \%\end{array}$ & & $6-12 \mathrm{keV}$ & & $6-12 \mathrm{ke}$ & \\
\hline$F L_{N T H}$ & $\mathrm{ph} / \mathrm{s} / \mathrm{cm}^{2} / \mathrm{keV}$ & - & 66.9 & - & 289.5 \\
\hline$F L_{T H}$ & $\mathrm{ph} / \mathrm{s} / \mathrm{cm}^{2} / \mathrm{keV}$ & - & 2710.0 & - & 11101.1 \\
\hline $\begin{array}{l}F L_{S U M} \\
\text { вох }\end{array}$ & $\mathrm{ph} / \mathrm{s} / \mathrm{cm}^{2} / \mathrm{keV}$ & $3043.8-3155.2$ & 2788.3 & $551.1-503.2$ & 11402.9 \\
\hline$F L_{S U M}$ & $\begin{array}{c}\mathrm{ph} / \mathrm{s} / \mathrm{cm}^{2} / \mathrm{keV} \\
\operatorname{arcsecs}\end{array}$ & 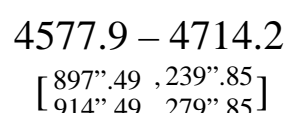 & $\begin{array}{c}2788.3 \\
-\end{array}$ & 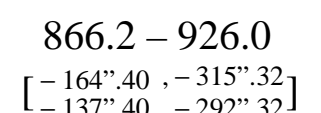 & $\begin{array}{c}11402.9 \\
-\end{array}$ \\
\hline$F_{B K G}$ & $\begin{array}{c}\mathrm{ph} / \mathrm{s} / \mathrm{cm}^{2} / \mathrm{keV} \\
\operatorname{arcsecs}\end{array}$ & 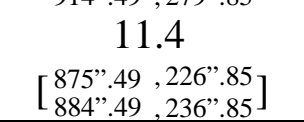 & $\begin{array}{l}- \\
-\end{array}$ & $\begin{array}{c}12.3 \\
{\left[\begin{array}{c}-175^{\prime \prime}, 40,-334^{\prime \prime}, 32 \\
-16, * 40,-325^{\prime}, 32\end{array}\right]}\end{array}$ & $\begin{array}{l}- \\
-\end{array}$ \\
\hline
\end{tabular}

$F P_{1}$ and $F P_{2}$ - flux measured at footpoints; $F P_{A V E}$ - average flux from $F P_{1}$ and $F P_{2} ; F_{B K G}$ - background flux;

$F L_{N T H}$ and $F L_{T H}$ - flux of non-thermal and thermal emissions from entire flare's loop; $F L_{S U M}$ - the total flux coming from the entire flare's loop. 
calculated fluxes added value of the background was derived from the RHESSI image $\left(F_{B K G}\right)$ using the box defined as coordinates of the corners (see Table 2). For the lowest energy range (6-12 keV) the applied methodology is more complicated. In this energy range and at the time when the images were registered for the analysed flares the thermal component dominates, although the non-thermal emission adds a small contribution (see Figures 12 and 13). Therefore, calculated emission of the model in this energy range should be the sum of the thermal $\left(F L_{T H}\right)$ and non-thermal $\left(F L_{N T H}\right)$ emissions and such value $\left(F L_{S U M}\right)$ is compared with the observations. The modelled emission was integrated over the entire loop, and also in this case the value of the background is added and derived from the RHESSI image. The only observed structure of the RHESSI images in this energy range is the flare's loop. As in the case of a high energy range the same two methods of estimation of the areas of loop's emission were used.

For the analysed event of June $2^{\text {nd }}, 2002$ in the 30-80 keV energy range emission from the model is about 2 times bigger than observed flux within an isophote of $30 \%$ and is outside the range of errors. A similar situation occurs for the observed flux using a box method, where the obtained the synthesized emission is $30 \%$ higher than the emission derived from the observation. A much larger inconsistency occurs for the 6-12 keV energy range. In this case the emission calculated from the model is about 20 times greater within an isophote of $30 \%$ and about 12 times greater using the box than the observed emissions, respectively. The obtained result generally agrees with the relations of the synthesized and observed total fluxes shown in Figure 12 for the selected time. Results of photometry of the June $2^{\text {nd }}, 2002$ solar flare show that the synthesized emission in the $35-45 \mathrm{keV}$ energy range is about 4 times lower than the observed emission within an isophote of $30 \%$. Within the defined box this ratio is about 7. Good agreement was obtained for the 6-12 $\mathrm{keV}$ energy range, where emission from the model is about $11 \%$ lower for an isophote of $30 \%$, and about $60 \%$ lower for the box than the observed emission. As for the first analysed event, the received result agrees with the relations of the synthesized and observed total fluxes shown in Figure 13 for the selected time.

\section{Discussion \& Conclusions}

The modelling of the two solar flares observed on February $20^{\text {th }}, 2002$ and June $2^{\text {nd }}, 2002$ was conducted under the assumption that only non-thermal electron beams delivered energy to the flaring loop via the Coulomb collisions and the NTEs heating function was calculated based on the parameters derived from observed HXR spectra. Comparison of modelling results with observations shows that SXR and HXR emissions of both single-loop like events could be fully explained by electron beam-driven evaporation only. There is no need for any additional heating mechanism to explain the observed SXR and HXR emissions and dynamics of the flaring plasma. 
The use of the Fokker-Planck formalism allowed for an improvement of the calculated models, if compared with previous works, by direct comparison of the observed and modelled distributions of the HXR emission. The best compatibility of the model and the observations was obtained for the June $2^{\text {nd }}, 2002$ event, both the 0.5-4 $\AA$ GOES light curve and for total fluxes in $50-100 \mathrm{keV}$, 25-50 keV, 12-25 keV and 6-12 keV energy band. Poorer concordance compared to the first event is obtained for the February $20^{\text {th }}, 2002$ solar flare. In both cases, the largest differences between the observed and synthesized light curves appeared in the 12-25 keV energy range. This difference is mainly caused by a thermal emission component which gives too small a contribution in this range to the total flux. In higher ranges of energy, differences between the model and the observed fluxes decrease. In these ranges the non-thermal component dominates. This behaviour appears to be justified because, even if the plasma distribution in the model does not correspond to the observation, the non-thermal electrons which have high energies can penetrate deeper into the chromosphere and do not interact with matter during their travel in contrast to the low energy non-thermal electrons that convey energy in the higher parts of the flare loop. The HXR non-thermal emission is directly related to the flux of the accelerated NTEs whereas the SXR and HXR thermal emissions are related to the energy deposited by the same NTEs flux. Another important factor influencing the differences between the modelled and observed fluxes appearing in the 12-25 keV energy range is the assumption that injected electrons have a power law energy spectrum. As shown by Liu. Petrosian, \& Mariska (2009) using the Fokker-Planck model of particle transport and the injected electron spectrum based on stochastic acceleration, higher coronal temperatures and densities, larger upflow velocities, and faster increases of these quantities are obtained than in the model where electron injection spectrum was of power law. This is due to the fact that the injected electron spectrum smoothly spans from a quasi-thermal component to a non-thermal tail. The injected beam of electrons contains a lot of low energy electrons which deposit their energy primarily in the corona and indirectly cause stronger evaporation, and thereby the thermal emission component could give a greater contribution to the total flux in the 12-25 $\mathrm{keV}$ energy range. An underestimation of the thermal component in the model may also probably indicates inconsistencies between distributions of the mass in real flaring loops and in their models. This wrong distribution of mass in the model may arise by relative simplicity of the applied numerical 1D code, coarse estimation of the geometrical loop parameters, errors in RHESSI spectra restoration and beam parameters obtained from fitting NTEs, possible problems in a GOES's calibration and by using an analytical formula for NTEs heating function. Additionally, it is assumed that at the beginning we have an initial, quasi-stationary preflare model of the flaring loop with the mass distribution consistent with the observations and begins to heat the plasma using NTEs heating function which was calculated based on the parameters derived from observed HXR spectra. These parameters are determined by fitting RHESSI spectra and vitiated by errors. The parameters uncertainties reported by OSPEX can give some idea of the stability of the fit results. They do not account for interdependence between fit parameters (e.g. between the temperature 
and emission measure values of an isothermal emission component or the power-law index of the electron energy distribution, and low energy cut-off of the electron distribution for the non-thermal component) and can often underestimate the true uncertainties when the chi-squared space is not well-behaved (e.g. when it is degenerate along one or more dimensions). Additionally, an influence of the thermal component on the spectra can be a source of errors of the derived parameter of electrons' distribution. A detailed analysis of this type of relations and their influences on the derived parameters of the fitted spectra was conducted by Saint-Hilaire \& Benz (2005). In addition, the use of analytical formula for NTEs heating function, which is only an approximation, may introduce additional errors into the model. However, taking into account the results published by Liu, Petrosian. \& Mariska (2009) that the model with analytical approximation of heating gives about $10 \%$ difference only, compared to the model with a Fokker-Planck regime, this is an acceptable approximation especially when we consider the benefits of the speed of calculation and all the other errors arising from the simplifications used in the model. As a result of all these factors the energy will be deposited in different layers of the flare model compared to the real event. This may lead to receiving other physical parameters of the plasma evolution in the loop model than calculated from observations, particularly the distribution of mass in the flaring loop. This causes the previously described differences between the observed and synthesized light curves. One can also assume that at the beginning, an initial, quasi-stationary preflare model of the flaring loop with mass distribution inconsistent with the observations which may be caused by poorly determined geometrical parameters. In this case, even the use of the parameters derived from observed HXR spectra without errors and "ideal" NTEs heating function will lead to the same effects as in the first case. In reality, however, the analytical heating function is used (with all its consequences) and its parameters with errors. Similarly the results of photometry for a high energy range show that the best compliance was obtained for the June $2^{\text {nd }}, 2002$ flare where the synthesized emission in the best case is only $30 \%$ higher than the emissions measured from the observation. For the February $20^{\text {th }}, 2002$ flare, synthesized emission is about 4 times lower than the observed one. In the low energy range the best conformity was obtained for the February $20^{\text {th }}$, 2002 flare, where emission from the model is only about $11 \%$ lower than the observed one. The larger inconsistency occurs for the June $2^{\text {nd }}, 2002$ solar flare, where synthesized emission in the best case is about 12 times more than emission from observation. The observed differences which are evident on conducted photometry are primarily caused by the assumption that the model of the flare is symmetric and there are no differences in the emissions originating from the feet of the flare's loop. In fact, as has been demonstrated already, the observations indicate that this condition is not preserved (see Table 2). The obtained results are also affected by all the factors that were presented in an earlier discussion.

For instance the obtained errors are similar to results from previous work (Falewicz. Siarkowski. \& Rudawy 2011) and for the non-thermal fits in the decay phase had small formal errors of fitting parameters 
(about $1 \%$ for $\delta$ and $\mathrm{E}_{\mathrm{c}}$ ), while the statistical errors were of the order of $15-25 \%$ for $\delta$ and $11-19 \%$ for $E_{c}$. The error ranges were determined for each of the analysed events. The problem of errors may be particularly important for the very early and decay phases of a solar flare where the spectra registered by RHESSI could also be reasonably fitted using a thermal model only with an acceptably low value of $\chi^{2}$ estimator. In these cases, the temperature values obtained were higher than the temperature calculated from the GOES light curves at the same time. For the early phase this difference is so high that almost certainly a non-thermal component in the spectra occurred. This situation is not so clear for the decay phase. Differences of temperature between the RHESSI and GOES are not so large, and amount to about 2-3 MK only. In this phase of the flare the thermal component dominates and covers the weak non-thermal component in the spectra. The argument for the occurrence of non-thermal emission at this stage of the flare can be extended only on the basis of the model where during the decay phase heating is necessary (see the green dotted curve in Figure 8 and 10 (upper left panel) representing the evolution of the plasma in the flaring loop without heating). Another argument might be an idea presented by Brosius \& Holman (2009) assuming that during this phase non-thermal emission can be below RHESSI's detection threshold.

Despite all these problems, flare modelling presented in this paper simulates the main physical processes in the right way. It is worth stressing that the HD-1D codes are sufficient in modelling of the flaring events occurring in simple, single-loop like configurations of the magnetic field, even in such elaborated applications like a synthesis of the BCS spectra (Falewicz. Rudawy. \& Siarkowski 2009b). Comparison of the synthesized and observed spectra confirm that the distribution of matter and the velocities in the flaring loop agree roughly with the observed values.

In summary, as shown in our previous papers (Siarkowski, Falewicz, \& Rudawy 2009; Falewicz, Siarkowski, 2011), efficient heating of the flaring plasma by non-thermal electron beams starts in the very early phase of the flare, significantly before the impulsive phase, and lasts a long time during its gradual phase. This heating is so efficient that no other heating mechanism is necessary to explain emitted SXR and HXR fluxes. In particular, during the gradual phase of the flare a much slower decay of the SXR flux is observed than could be expected from an analytical estimation of energy losses of the flaring plasma by radiation and by conduction (Cargill. Mariska, \& Antiochos 1995) or from HD numerical modelling of the flares (for example: Reale et al. 1997). However, the SXR decay time of the solar flares is usually much extended if compared with the previously mentioned cooling times, proving that during this phase of the flares significant heating is also present. Similar conclusions have already been shown in papers by McTiernan et al. (1993) and Jiang et al. (2006). As demonstrated in the present work for the analysed $20^{\text {th }}$ February, 2002 and $2^{\text {nd }}$ June, 2002 flares all of the energy necessary for heating the flaring loops during all phases of the events was supplied by the NTEs. However, please note that this does not preclude the existence of other, secondary sources of heating or mechanisms of energy transfer. 
During modelling the flares, the light curves for a number of SXR and HXR energy ranges were successfully reproduced. The use of the extended code with the Fokker-Planck formalism allowed for an improvement of the calculated models and made it possible to ascertain which components of the emission (thermal or non-thermal) play a dominant role in the chosen energy range. However, taking into account the results of photometry, no doubt a significant refinement of the applied numerical models and more sophisticated implementation of the various physical mechanisms involved are required to achieve a better agreement.

\section{Acknowledgments}

The author acknowledges the RHESSI, and SOHO consortia for the excellent data and is grateful to an anonymous referee for constructive comments and suggestions, which have proved to be very helpful in improving the manuscript. The research leading to these results has received funding from the European Community's Seventh Framework Programme ([FP7/2007-2013]) under grant agreement $\mathrm{n}^{\circ}$ [606862].

\section{REFERENCES}

Antonucci, E., Gabriel, A. H., \& Dennis, B.R. 1984, ApJ, 287, 917

Aschwanden, M. J., Fletcher, L., Sakao, T., Kosugi, T., \& Hudson, H. 1999, ApJ, 517,977

Aschwanden, M. J., Brown, J. C., \& Kontar, E. P. 2002, Sol. Phys., 210, 383

Aschwanden, M. J. 2005, Physics of the Solar Corona (Berlin: Springer Praxis Books)

Bai, T., \& Ramaty, R. 1978, ApJ, 219, 705

Brosius, J. W., \& Holman, G. D. 2009, ApJ, 692, 492

Brown, J.C. 1971, Sol. Phys., 18, 489

Cargill, P. J., Mariska, J. T., \& Antiochos, S. K. 1995, ApJ, 439, 1034

Carovillano, R. \& McClay, J. F. 1968, Physics of the Magnetosphere (New York: Reinhold), p. 534

Delaboudinier̀e, J. P., Artzner, G. E., Brunaud, J., et al. 1995, Sol. Phys., 162, 291 
Dere, K. P., Landi, E., Mason, H. E., Monsignori-Fossi, B. C.,\& Young, P. R. 1997, A\&A, 125, 149

Donnelly, R. F., Grubb, R. N., \& Cowley, F. C. 1977, NOAA Tech. Memo. ERL SEL-48

Emslie, A. G. 1978, ApJ, 224, 241

Falewicz, R., Rudawy, P., \& Siarkowski, M. 2009a, A\&A, 500, 901

Falewicz, R., Rudawy, P., \& Siarkowski, M., 2009b, A\&A 508, 971

Falewicz, R., Siarkowski, M., \& Rudawy, P., 2011, ApJ 733, 37

Fisher, G. H. 1989, ApJ, 346, 1019

Fisher, G. H., Canfield, R. C., \& McClymont, A. N. 1985a, ApJ, 289, 414

Fisher, G. H., Canfield, R. C., \& McClymont, A. N. 1985b, ApJ, 289, 425

Fisher, G. H., Canfield, R. C., \& McClymont, A. N. 1985c, ApJ, 289, 434

Grigis, P. C., \& Benz, A. O. 2004, A\&A, 426, 1093

Guo, J., Liu, S., Fletcher, L., \& Kontar, E. P. 2011, ApJ, 728, 4

Holman, G. D., Sui, L., Schwartz, R. A.,\& Emslie, A. G. 2003, ApJ, 595, L97

Hurford, G. J., Schmahl, E. J., Schwartz, R. A., et al. 2002, Sol. Phys., 210, 61

Jakimiec, J., Sylwester, B., Sylwester, J., Serio, S., Peres, G., \& Reale, F. 1992, A\&A, 253, 269

Jiang, Y. W., Liu, S., Liu, W. \& Petrosian, V. 2006, ApJ, 638, 1140

Koch, H. W. \& Motz, J. W. 1959, Rev. Mod. Phys., 31, 920

Krucker, S., \& Lin, R. P. 2002, Sol. Phys., 210, 229

Landi, E., Del Zanna, G., Young, P. R., Dere, K. P., Mason, H. E., \& Landini M. 2006, ApJS, 162, 261

Leach, J. \& Petrosian, V. 1981, ApJ, 251,781

Lin, R. P., Dennis, B. R., Hurford, G. J., et al. 2002, Sol. Phys., 210, 3

Liu, W., Petrosian, V., \& Mariska, J. T. 2009, ApJ, 702, 1553

MacNeice, P., McWhirter, R.W.P., Spicer, D.S., \& Burgess, A. 1984, Sol. Phys., 90, 357 
Mariska, J. T., Boris, J. P., Oran, E. S., Young, T. R. Jr., \& Doschek, G. A. 1982, ApJ, 255, 738

Mariska, J. T., \& Poland, A. I. 1985, Sol. Phys., 96, 317

Mariska, J. T., Emslie, A. G., \& Li, P. 1989, ApJ, 341, 1067

Mazzotta, P., Mazzitelli, G., Colafrancesco, S., \& Vittorio, N. 1998, A\&AS, 133, 403

McTiernan, J. M., \& Petrosian, V. 1990, ApJ, 359, 524

McTiernan, J. M., Kane, S. R., Loran, J. M., et al. 1993, ApJ, 416, L91

Meshalkina, N. S., Uralov, A. M., Grechnev, V. V., Altyntsev, A. T., \& Kashapova, L. K. 2009, PASJ, 61, 791

Metcalf, T.R., Hudson, H.S., Kosugi, T., Puetter, R. C., \& Pina, R. K. 1996, ApJ, 466, 585

Nagai, F., \& Emslie, A.G. 1984, ApJ, 279, 896

Peter, H. \& Bingert, S. 2012, A\&A, 548, A1

Reale, F., Betta, R., Peres, G., Serio, S., \& McTiernan, J. 1997, A\&A, 325, 782

Reep, J. W., Bradshaw, S. J., \& McAteer, R. T. J. 2013, ApJ, 778, 76

Saint-Hilaire, P., \& Benz, A. O. 2005, A\&A, 435, 743

Schmelz, J.T., Holman, G.D., Brosius, J.W., \& Willson, R. F. 1994, ApJ, 434, 786

Serio, S., Reale, F., Jakimiec, J., Sylwester, B., \& Sylwester, J. 1991, A\&A, 241, 197

Siarkowski, M., Falewicz, R., \& Rudawy, P. 2009, ApJL, 705, L143

Smith, D. M., Lin, R. P., \& Turin, P. et al. 2002, Sol. Phys., 210, 33

Sui, L., Holman, G. D., Dennis, B. R., Krucker, S., Schwartz, R. A., \& Tolbert, K. 2002, Sol. Phys., 210,245

Sui, L., Holman, G. D., \& Dennis, B. R. 2006, ApJ, 646, 605

Sui, L. H., Holman, G. D., \& Dennis, B. R. 2007, ApJ, 670, 862

Sui, L., Holman, G. D., \& Dennis, B. R. 2008, AdSpR, 41, 976

Tandberg-Hanssen, E., \& Emslie, A. G. 1988, The physics of solar flares (Cambridge University Press) 
Veronig, A. M., Brown, J. C., Dennis, B. R., Schwartz, R. A., Sui, L., \& Tolbert, A. K. 2005, ApJ, 621, 482

Warmuth, A., Holman, G. D., Dennis, B. R., Mann, G., Aurass, H., \& Milligan, R. O. 2009a, ApJ, 699, 917

Warmuth, A., Mann, G., \& Aurass, H. 2009b, A\&A, 494, 677

Warren, H. P. 2006, ApJ, 637, 522 